Cahiers $d u$ MONDE RUSSE

\section{Cahiers du monde russe}

Russie - Empire russe - Union soviétique et États indépendants

\title{
« Kto naš, kto ne naš » Théorie et pratiques de la citoyenneté à l'égard des populations conquises
}

Le cas des Polonais en URSS, 1939-1946

\section{Catherine Gousseff}

\section{(2) OpenEdition}

\section{Journals}

Édition électronique

URL : https://journals.openedition.org/monderusse/8621

DOI : 10.4000/monderusse. 8621

ISSN : $1777-5388$

\section{Éditeur}

Éditions de l'EHESS

Édition imprimée

Date de publication : 1 avril 2003

Pagination : 519-558

ISBN : 2-7132-1813-6

ISSN : $1252-6576$

\section{Référence électronique}

Catherine Gousseff, « « Kto naš, kto ne naš » Théorie et pratiques de la citoyenneté à l'égard des populations conquises », Cahiers du monde russe [En ligne], 44/2-3 | 2003, mis en ligne le 01 janvier 2007, consulté le 02 septembre 2022. URL : http://journals.openedition.org/monderusse/8621 ; DOI https://doi.org/10.4000/monderusse.8621 


\section{CAIR N}

chercher : repérer : avancer

Cet article est disponible en ligne à l'adresse :

http://www.cairn.info/article.php?ID REVUE=CMR\&ID NUMPUBLIE=CMR 442\&ID ARTICLE=CMR 4420519

« Kto nas, kto ne nas » Théorie et pratiques de la citoyenneté à l'égard des populations conquises. Le cas des Polonais en URSS, 1939-1946

par Catherine GOUSSEFF

\section{| Editions de l'EHESS | Cahiers du monde russe}

\section{3/2-3 - Vol 44}

ISSN 1252-6576 | ISBN 2713218136 | pages 519 à 558

Pour citer cet article :

- GOUSSEFF C., «Kto nas, kto ne nas » Théorie et pratiques de la citoyenneté à l'égard des populations conquises. Le cas des Polonais en URSS, 1939-1946, Cahiers du monde russe 2003/2, Vol 44, p. 519-558.

Distribution électronique Cairn pour les Editions de l'EHESS.

(C) Editions de l'EHESS. Tous droits réservés pour tous pays.

La reproduction ou représentation de cet article, notamment par photocopie, n'est autorisée que dans les limites des conditions générales d'utilisation du site ou, le cas échéant, des conditions générales de la licence souscrite par votre établissement. Toute autre reproduction ou représentation, en tout ou partie, sous quelque forme et de quelque manière que ce soit, est interdite sauf accord préalable et écrit de l'éditeur, en dehors des cas prévus par la législation en vigueur en France. Il est précisé que son stockage dans une base de données est également interdit. 


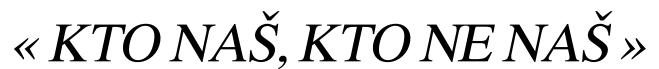 THÉORIE ET PRATIQUES DE LA CITOYENNETÉ À L'ÉGARD DES POPULATIONS CONQUISES
}

\author{
Le cas des Polonais en URSS, 1939-1946
}

La conception administrative de l'identité, telle qu'elle apparaît au début des années 30 lors de l'élaboration des premiers passeports intérieurs soviétiques et son rôle dans les pratiques d'enregistrement, en tant qu'instrument de contrôle et d'épuration sociale, ont fait l'objet d'un renouveau historiographique important ${ }^{1}$. Ces travaux ont montré l'incidence de la contrainte administrative dans les déplacements forcés de population et, plus généralement, dans les critères de stigmatisation qu'elle a contribué à fixer, voire à étendre, dans le cours de la décennie. L'appartenance et l'origine sociales des individus, facteurs essentiels de reconnaissance ou d'exclusion dans les premières actions de passeportisation des grands centres urbains, se sont combinées de façon progressive à cet autre déterminant de l'identité que fut la nationalité ou l'appartenance ethnique, notamment lors de l'extension des opérations d'enregistrement aux régions multiculturelles des territoires frontaliers. L'analyse de cette évolution majeure qu'a représentée l'ethnicisation croissante du regard porté par l'État sur la société complète, sous un autre angle, les travaux engagés sur les grandes vagues de répressions «nationales » de la seconde moitié de la décennie, qui ont touché en particulier

\footnotetext{
1. N. Moine, « Passeportisation, statistique des migrations et contrôle de l'identité sociale », Cahiers du Monde russe, 38, 4, 1997, p. 587-600 ; id., « Le système des passeports à l'époque stalinienne. De la purge des grandes villes au morcellement du territoire, 1932-1953», Revue d'Histoire moderne et contemporaine, 1, 2003, p. 145-169; G. Kessler, « The passport system and state control over population flows in the Soviet Union, 1932-1940 », Cahiers du Monde russe, 42, 2-4, 2001, p. 477-504 ; D. Shearer, « Social disorder, mass repression and the NKVD during the $1930 \mathrm{~s} »$, ibid., p. 505-534.
} 
les Polonais et les Allemands des régions occidentales et les Coréens des régions orientales de l'URSS'2.

Cet article s'inscrit dans le prolongement des problématiques ouvertes sur la conception et l'usage des déterminants de l'identité. Il les aborde dans la période de la guerre, en se concentrant, cependant, sur la citoyenneté, objet qui a été jusqu'à maintenant peu exploré. La citoyenneté a revêtu une évidente actualité lors de la conquête en 1939 des territoires orientaux de la Pologne et de la soviétisation des populations qui a suivi. Quelle conception les autorités soviétiques ont-elles eue de l'appartenance étatique et sur quels fondements l'ont-elles appliquée ? Cette question est analysée à partir du cas de quelque 260000 « anciens citoyens polonais », originaires de Pologne centrale et des territoires annexés, qui, pour avoir été déportés vers l'Est en 1940-1941, n'ont pas connu l'occupation allemande et ont, en revanche, vécu toute la guerre sur le territoire soviétique avant, finalement, d'être évacués vers la Pologne à l'issue du conflit. Leur sujétion - polonaise ou soviétique - , plusieurs fois remise en cause en fonction de l'état des relations bilatérales, a reposé sur des critères changeants. D'abord conçue en fonction de la territorialisation des individus, la citoyenneté a, par la suite, été définie selon leur appartenance ethnique, cette donnée de l'identité devenant alors centrale dans les pratiques d'enregistrement visant à établir les statuts. Elle fournit de ce point de vue un éclairage particulier sur l'utilisation de l'ethnicité comme fondement de la légitimité étatique d'une part, et d'autre part comme instrument au service des modes d'assignation identitaires. Dans un troisième temps, enfin, la citoyenneté a été conçue en fonction de l'ancienne appartenance étatique et l'identité des personnes a été validée en fonction des preuves documentaires exigées de leur précédente sujétion. L'extrême variation des critères en jeu dans les modalités d'attribution de la citoyenneté souligne l'absence de définition fixe de cette notion, ou en tout cas son caractère confus. La citoyenneté est surtout apparue, s'agissant des Polonais déportés, comme un objet politique aux mains des autorités soviétiques, elle fut abordée de façon essentiellement instrumentale dans un contexte bilatéral qui connut plusieurs bouleversements : rompues en 1939, rétablies en 1941 lors du passage de l'URSS dans le camp allié, les relations diplomatiques polonosoviétiques ont été dissoutes une fois encore en avril 1943 avant d'être reformulées avec des interlocuteurs nouveaux à partir de l'été 1944.

L'objectif de cette étude est d'abord de retracer l'usage de la citoyenneté telle qu'elle apparaît dans la succession des différentes séquences précitées, en analysant les logiques politiques à l'œuvre dans l'énonciation des principes et leur traduction dans les modalités d'application de la sujétion. Ce dernier aspect renvoie aux pratiques d'assignation identitaire, où l'exigence de preuve documentaire a fait apparaître, notamment, le rôle des médiateurs extérieurs. L'administration de l'identité conduit, en effet, à analyser les modalités de gestion des Polonais en

2. Pour une rétrospective de ces répressions, voir T. Martin, The affirmative action empire. Nations and nationalism in the Soviet Union, 1923-1939, Ithaca - Londres, Cornell University Press, 2001 (chap. 8, p. 309-343). 
URSS non seulement lorsqu'ils furent officiellement représentés par l'ambassade polonaise, rétablie dans ses fonctions entre 1941 et 1943, mais également par la suite. La création de l'Union des patriotes polonais (Związek patriotów polskich), organisation étroitement liée au parti communiste d'Union soviétique, relaya leur prise en charge à partir de 1943. Cette configuration particulière s'inscrit dans le champ encore mal connu des groupes de médiation qui ont été créés en URSS au sein de populations spécifiques, comme les réfugiés espagnols ou les prisonniers de guerre, et qui, lorsqu'ils ont été abordés, l'ont surtout été dans le prisme de l'inféodation politique au régime soviétique et non dans les formes de négociation, voire dans les compromis, que leur intervention a rendu possibles.

L'activité de l'Union des patriotes polonais à l'égard des « anciens citoyens polonais » pose, parallèlement aux opérations successives d'enregistrement, mais de façon contradictoire, la question de l'intention politique dans la destinée de ce groupe. Entre la volonté d'intégration, manifestée à travers la campagne de (re)passeportisation de 1943, et les évacuations effectives de 1946 vers la Pologne, s'ouvrent plusieurs interrogations sur le sens des décisions politiques et le rôle que joue en amont l'action administrative dans leurs orientations.

\section{Conquérir et réprimer : le pouvoir soviétique face à ses nouveaux citoyens}

Les vingt et un mois qui séparent le franchissement par l'Armée rouge des frontières orientales de la Pologne, le 17 septembre 1939, de la pénétration en juin 1941 des troupes de la Wehrmacht dans les territoires incorporés à l'URSS, représentent l'une des périodes les plus sensibles et les plus douloureuses des relations polonosoviétiques au Xxe siècle. Réalisée dans le cadre du Pacte germano-soviétique, l'annexion des régions orientales de la Pologne n'a pas seulement été la manifestation la plus spectaculaire de la communauté d'intérêts qui, au-delà de leurs divergences idéologiques, a ligué Hitler et Stalin durant les deux premières années de la guerre. Le « quatrième partage », ainsi que les Polonais ont pris coutume de désigner l'événement, par référence aux morcellements successifs de leur pays au $\mathrm{XVIII}^{\mathrm{e}}$ siècle $(1772,1793,1795)$, ou la première occupation soviétique, comme le dénomment encore certains historiens polonais, est une période qui a longtemps contenu de nombreuses «taches blanches », avant de faire l'objet de multiples introspections historiographiques depuis l'effondrement du bloc soviétique et l'ouverture des archives qui l'a accompagné ${ }^{3}$. Ces travaux récents éclairent les

3. C'est en Pologne surtout que le développement des travaux sur la période a été le plus important, se focalisant sur les différents aspects de l'agression soviétique (intervention de l'Armée rouge, arrestations et détentions des militaires et des civils, assassinat des officiers polonais et des détenus civils au printemps 1940, déportations). Voir l'état des lieux historiographique dressé par S. Ciesielski, W. Materski, A. Paczkowski, Represje sowieckie wobec Polaków i obywateli polskich, Varsovie, Karta, 2000. La contribution des historiens russes sur la période concerne, elle, principalement les déportations (outre les travaux de N. F. Bugaj et de P. Poljan sur les déportations des peuples, en particulier des Polonais, voir plus spécifiquement V. S. Pasardanova, « Deportacija naselenija iz Zapadnoj Ukrainy i Zapadnoj Belorusii v 1939-1941 gg. » (La dépor- 
différentes stratégies mises en oeuvre dans l'entreprise de soviétisation visant, selon l'expression de l'historien Amir Weiner, «à réaliser vingt ans en deux ans ${ }^{4}$ dans la mesure où l'ambition de la direction stalinienne était, en effet, de conformer sous tous les aspects - politiques, économiques et sociaux - les nouveaux territoires aux républiques soviétiques d'Ukraine et de Biélorussie auxquelles ils se trouvaient désormais rattachés. La radicalité de cette entreprise s'est exprimée sous la forme de différentes offensives à l'encontre de la société, qui se sont succédé sans relâche jusqu'à l'invasion allemande, faisant de la dimension répressive de la soviétisation l'une des principales caractéristiques de la période.

Dans un premier temps, la situation de ces régions occupées fut assez spécifique dans le sens où les conflits qui l'ont marquée n'ont pas été, sinon dans une moindre mesure par rapport aux étapes ultérieures, le fait direct des nouvelles autorités. Alors que l'armée soviétique n'a pas eu à faire face à d'importantes confrontations (moins de 3000 victimes dans ses rangs), un déferlement de violences sociales a en revanche accompagné l'arrivée des forces armées, sous l'effet conjugué de la vacance effective du pouvoir, en particulier dans les campagnes, et des actions d'incitation à la haine socio-ethnique menées par les nouvelles autorités à l'égard des anciens maîtres polonais («polskie pany ») . Dans une région où la tutelle de l'État polonais avait été largement contestée dès le début des années 1920, où la population polonaise y était minoritaire tout en ayant été sur-représentée aux postes-clés de l'économie et des administrations locales, l'instigation à la revanche de classes réactivait dans une même dynamique les antagonismes sociaux et ethniques, visant en premier lieu les Polonais ${ }^{6}$. Cette stigmatisation laissa des traces durables dans la minorité polonaise qui se sentit agressée durant toute la période du seul fait de son identité, même si les répressions à venir allaient toucher l'ensemble de la population des territoires conquis, officiellement incorporés à l'URSS les $1^{\text {er }}$ et 2 novembre 1939.

Bien que les interpellations et les détentions soient devenues des pratiques courantes à partir de septembre 1939, la mise en place d'une politique d'épuration à

tation de la population d'Ukraine occidentale et de Biélorussie occidentale en 1939-1941), Novaja i novejšaja istorija, 2, 1989, p. 25-40 ; A. E. Gur' janov, ed., Repressii protiv Poljakov i pol'skih graždan (La répression contre les Polonais et les citoyens polonais), Moscou, NIPC Memorial, 1997. Les sources polonaises issues du gouvernement de Londres ont également fait l'objet, depuis les années 1980, de nouvelles investigations qui ont fourni, sous certains aspects, un éclairage complémentaire à l'exploitation des archives soviétiques. Voir, à ce propos, J. T. Gross, Revolution from abroad. The Soviet conquest of Poland's Western Ukraine and Western Bielorussia, Princeton - Oxford, Princeton University Press, 1988 (rééd. 2002); K. Sword, ed., The Soviet takeover of the Polish Eastern provinces, New York, St. Martin Press, 1991.

4. A. Weiner, « Wild West, window to the West: The Soviet western frontier, 1939 to present », communication présentée au Centre d'études du monde russe, soviétique et post-soviétique de l'EHESS, Paris, 15 novembre 2002.

5. J. T. Gross, Revolution from abroad..., op. cit., p. 17-69 ; A. Głowacki, Sowieci wobec Polaków na ziemiach wschodnich II Rzeczypospolitej 1939-1941, Łódź, WUŁ, 1998, p. 39-63.

6. D'après le recensement polonais de 1931 (enregistrement des nationalités selon la langue maternelle), les Polonais représentaient $43 \%$ de la population totale des territoires annexés (Ukrainiens $-24,2 \%$, Biélorusses $-7,5 \%$, juifs $-8,8 \%$ ) et étaient très inégalement répartis selon les régions. 
grande échelle de la société date de la fin de l'année. Celle-ci fut conçue par le pouvoir central sous la forme de deux actions principales, de passeportisation d'une part, de déportation de l'autre. Leur réalisation a cependant donné lieu à un enchâ̂nement de mesures de plus en plus radicales qui atteignirent leur apogée en mars 1940, lorsque fut décidé, sur proposition de Berija, de procéder à la liquidation de près de 15000 officiers polonais prisonniers de guerre et de 11000 détenus civils 7 . Cette conjonction d'opérations constitue la grande singularité de la période qui s'étend sur les six premiers mois de l'année 1940 et qui représenta le « pic » de la terreur étatique déployée dans la région.

\section{La campagne de passeportisation}

À première vue, la décision prise par le Sovnarkom, le 29 novembre 1939, relative à la passeportisation des nouveaux territoires se situait dans la continuité du processus d'institutionnalisation de l'État soviétique, elle avait pour objectif de matérialiser la nouvelle sujétion des populations locales par la délivrance de documents d'identité. Cette action se référait, en effet, à la loi du 19 août 1938 « sur la citoyenneté de l'URSS », qui marqua tardivement l'unification des droits et des modalités d'acquisition de la citoyenneté soviétique.

Cette loi était novatrice à plusieurs égards ${ }^{8}$. Jusque-là en effet, la naturalisation n'avait été conçue que pour certaines catégories d'étrangers, qui devaient être soit des membres de la classe ouvrière et paysanne, soit des personnes jouissant du statut de réfugié, ou encore des étrangers mariés à des Soviétiques ${ }^{9}$. Désormais ces restrictions étaient abolies et pouvait accéder à la citoyenneté soviétique toute personne, indépendamment de sa race et de sa nationalité (au sens d'appartenance ethnique : nacional'nost'), qui en adressait personnellement la requête au Présidium du Soviet suprême de l'URSS ${ }^{10}$. Cette instance était également la seule habilitée à statuer sur la « sortie » de la citoyenneté (vyhod iz graždanstva) des étrangers

7. 14552 officiers furent effectivement assassinés et 7305 détenus civils. Katyń, Dokumenty Ludobójstwa. Dokumenty i materiały archiwalne przekazane Polsce 14.10.1992, Varsovie, PAN, 1992 (édition bilingue russo-polonaise), p. 42-44 pour les bilans.

8. Pour un aperçu de l'évolution de la législation en la matière, cf. V. S. Ševcov, Graždanstvo SSSR (La citoyenneté de l'URSS), Moscou, MR, 1980, p. 20-23. Ju. Fel’̌̌tinskij, K istorii našej zakrytosti. Zakonodatel'nye osnovy sovetskoj immigracionnoj i emigracionnoj politiki (Histoire de notre fermeture. Les fondements juridiques de la politique soviétique d'immigration et d'émigration), Moscou, Terra, 1991.

9. Ces différentes catégories d'étrangers furent définies dès 1918, y compris celle de « réfugié », la RSFSR ayant été le premier État à reconnaître l'existence juridique des réfugiés politiques. Mais le contenu donné à ce statut subit plusieurs modifications, puisqu'à l'origine il désignait tout individu persécuté pour des raisons politiques et/ou religieuses, alors qu'à la veille des années 1930 il ne visait plus que les combattants actifs pour la liberté. Voir Ju. Fel’štinskij, op. cit.

10. Article 3 de la loi du 19 août 1938, Sbornik dejstvujuščih dogovorov, soglašenij i konvencij zaključennyh SSSR s innostrannymi gosudarstvami (Recueil des traités, accords et conventions en vigueur conclus par l'URSS avec les États étrangers), Moscou, MID, 1955, vol. 10, p. 223 224. 
naturalisés, procédure qui, nous le verrons, donnera lieu, au terme de la guerre, à une vaste campagne de vérification des Polonais.

La loi de 1938 définissait également pour la première fois le statut d'apatride que les Soviétiques avaient contribué, dans les faits, à promouvoir de façon déterminante ${ }^{11}$. Par rapport à la définition en vigueur depuis la convention internationale de 1933 selon laquelle l'apatride désignait toute personne privée de la protection de son État d'origine, les autorités soviétiques adoptèrent une conception beaucoup plus pragmatique de ce statut qui concernait « tout individu se trouvant sur le territoire de l'URSS, n'étant pas citoyen soviétique et n'étant pas en possession de document attestant son appartenance à un État étranger » (art. 8). La situation d'apatride n'était donc abordée que sous l'angle des conséquences de la perte de protection étatique - l'absence de pièces d'identité - et non de ses causes. Sa formulation revenait à statuer sur le simple constat de non-possession de passeport, pratique qui devint effectivement la règle au cours de la guerre, où cette situation d'apatride allait, de fait, être assimilée à une situation illégale.

L'élaboration de la loi de 1938 ne fut liée à aucun contexte factuel précis, qu'il s'agît d'entrées massives d'étrangers en URSS, comme ce sera le cas près d'un an plus tard lors de l'arrivée des réfugiés espagnols, ou d'acquisition de nouveaux territoires. Elle s'inscrivait en revanche dans le cadre de la consolidation de la législation soviétique à laquelle la promulgation de la nouvelle constitution de 1936 avait donné une impulsion majeure, arrivant à point nommé, quinze mois plus tard, pour procéder à la soviétisation massive des nouvelles populations incorporées.

Bien que l'opération de passeportisation ait été officiellement conçue pour formaliser l'attribution de la citoyenneté soviétique, son objectif réel, les modalités de son déroulement et ses incidences ne différenciaient pas foncièrement cette action de celles menées depuis 1933 au sein de la société soviétique. Cette remarque s'applique d'abord à la conception générale de la passeportisation qui concernait, selon les directives du 30 décembre 1939, non pas l'ensemble du territoire mais les centres industriels et urbains ainsi que les lieux jugés stratégiques, telles les zones frontalières. Outre la population rurale, plusieurs catégories de personnes, comme les prisonniers de guerre et les civils en état d'arrestation, en furent exclues ${ }^{12}$. D'autre part, l'action de passeportisation ne fut pas une banale formalité d'enregistrement. La volonté de contrôle social et politique, avec sa cohorte de mesures directement répressives ou stigmatisantes, s'est ouvertement manifestée tout au long de la campagne, instaurant, au cours de l'hiver 1940, un véritable climat d'inquisition.

11. La RSFSR avait été, en effet, pionnière dans l'application du principe de déchéance de citoyenneté qu'elle promulga dès 1921 à l'égard des ressortissants russes à l'étranger n'ayant pas fait acte d'obédience au gouvernement de la Russie soviétique. En dénationalisant plusieurs centaines de milliers de citoyens, la RSFSR conduisit les États européens à élaborer, sous l'égide de la Société des Nations, le statut d'apatride. Sur le rôle décisif des réfugiés russes dans l'élaboration de ce premier statut, voir C. Klein-Gousseff, « Immigrés russes en France, 19001950. Contribution à l'histoire politique et sociale des réfugiés », thèse de doctorat, Paris, EHESS, 1996, p. 387-420.

12. A. Głowacki, Sowieci..., op. cit., p. 77-78. 
L'ukaz du Soviet suprême de l'URSS du 29 novembre 1939 sur la mise en application de la loi de 1938 précisa quelles étaient les catégories d'individus concernés, à savoir : 1) tous les anciens citoyens polonais se trouvant sur les territoires de Biélorussie et d'Ukraine occidentales lors de l'intégration officielle de ces régions à l'URSS (1 er et 2 novembre 1939$)^{13} ; 2$ ) tous les individus arrivés sur les dits territoires conformément aux accords germano-soviétique et soviéto-lituanien de $1939^{14}$; 3) tous les individus, anciens citoyens polonais, ayant été déchus de la citoyenneté soviétique en $1921^{15}$. La définition des ayants droit de cet ukaz prêtait à confusion dans la mesure où elle opérait des distinctions dans les situations des « anciens citoyens polonais », notamment entre ceux qui étaient présents sur le territoire et ceux qui étaient arrivés dans les contextes particuliers précités. Elle conduisait ainsi à s'interroger sur les mesures à prendre face à une autre catégorie de population non évoquée mais très importante qui était constituée de tous les réfugiés « spontanés » ayant fui la Pologne centrale au moment de l'invasion allemande et qui étaient majoritairement des juifs. Des demandes de précision furent adressées au Présidium du Soviet suprême, mais six mois plus tard Kalinin se contentait toujours de répondre en reformulant les mêmes catégories ${ }^{16}$.

Bien des aspects de la campagne de passeportisation, autant dans son déroulement que dans ses bilans, restent mal connus, mais il est avéré qu'elle fut d'abord entreprise en Biélorussie occidentale, région stratégique par sa proximité avec la frontière, où elle débuta, théoriquement du moins, le 15 février 1940 dans les villes de Bialystok et de Grodno, avant de s'étendre à l'Ukraine en mars où elle rencontra, semble-t-il, de plus grandes difficultés ${ }^{17}$. La responsabilité et la conduite de cette action revinrent aux organes du parti qui manquaient de personnel, ne disposaient pas d'infrastructures suffisantes et étaient peu préparés à remplir cette mission qui incombait traditionnellement au NKVD ${ }^{18}$.

13. GARF, f. 7523 , op. 10, d. 67,1.1-2.

14. L'accord germano-soviétique du 16 novembre 1939 définit le principe d'un échange de populations entre les deux zones d'occupation, l'URSS s'engageant à assurer, sur la base du volontariat, le transfert des populations germaniques d'Ukraine et de Biélorussie, et l'Allemagne, les populations ukrainiennes, biélorusses, russes et ruthènes résidant en Pologne. GARF, f. 7523, op. 10, d. 67, 1. 5-23. Il existe une version française de ce texte, présentée, avec les accords de Lausanne sur les transferts gréco-turcs, comme étant un modèle en la matière ; voir Les transferts internationaux de population, Paris, 1946. L'accord soviéto-lituanien du 10 octobre 1939 avait été passé lors de la brève intervention soviétique d'octobre 1939 au cours de laquelle la région de la Wilenszczyzna avait été remise aux Lituaniens.

15. En effet, de nombreux réfugiés de la guerre civile, puis de la famine de 1922, s'étaient installés dans les territoires limitrophes de l'URSS. Lors de la promulgation du décret sur la déchéance de citoyenneté des Russes se trouvant à l'étranger, le gouvernement polonais estimait à 250000 le nombre de réfugiés se trouvant sur son territoire. Voir C. Klein-Gousseff, « Immigrés russes... », op. cit., p. 155-156

16. GARF, f. 7523 ,op. 10,d.67,1.24.

17. S. G. Filippov, «Dejatel'nost' organov VKP(b) v zapadnyh oblastjah Ukrainy i Belorussii v 1939-1941 » (L'activité des organes du VKP(b) dans les régions occidentales d'Ukraine et de Biélorussie en 1939-1941), in A. E. Gur'janov, ed., Repressii protiv Poljakov..., op. cit., p. 56-57. L'auteur signale la très grande pauvreté des archives locales relatives à la campagne de passeportisation. 
Dans sa conception même, l'opération de passeportisation visait à hiérarchiser la population selon les profils et les trajectoires des individus. Elle prévoyait, en conséquence, la délivrance de différents types de documents, allant d'un passeport à une simple attestation d'identité valable trois mois, et en fonction desquels étaient définis les droits ou les non-droits de résidence. A priori, sur la base de la présentation de différents papiers, tels que la fiche d'état civil, les certificats de travail et d'habitation (ainsi que deux photographies), les résidents pouvaient obtenir un passeport d'une validité maximale de trois ans (la norme ayant été alors de cinq ans dans le reste de l'URSS) ${ }^{19}$. L'obtention du passeport ne revenait pas cependant à accorder systématiquement le droit de résidence dans les lieux où il avait été délivré. Il pouvait comprendre des paragraphes restrictifs et en particulier le paragraphe 11 qui signifiait l'expulsion pure et simple du lieu de résidence au-delà d'un rayon de 100 kilomètres, pratique qui fut alors très répandue dans les principales villes d'Ukraine occidentale dans une période où l'on assistait, par ailleurs, à l'arrivée d'un nouveau personnel administratif, en provenance des régions orientales des républiques, qu'il fallait loger ${ }^{20}$. Le paragraphe 11 fut appliqué à différentes catégories de personnes, dont les familles de ceux qui se trouvaient en état d'arrestation. Les réfugiés, alors estimés à environ 300000 personnes, qui s'étaient massivement concentrés dans les villes occidentales furent également parmi les plus exposés à cette restriction ${ }^{21}$. Ils furent nombreux à devoir quitter à nouveau leur précaire abri pour aller plus à l'est ${ }^{22}$.

Outre les stigmatisations et les déplacements forcés qu'elle entraîna, l'opération de passeportisation donna lieu à de forts mouvements de résistance. Compte tenu du caractère volontaire et individuel de l' « entrée » dans la citoyenneté soviétique, l'enregistrement ne consistait pas en une simple démarche administrative, il impliquait une confrontation directe avec les autorités qui ressemblait bien souvent à un véritable interrogatoire sur les trajectoires, les affiliations politiques, la nationalité (ethnique) des individus. Cet élément de l'identité, consigné dans les documents soviétiques et théoriquement enregistré selon le principe de l'autodéclaration, fit, selon des témoignages a posteriori, l'objet de fortes pressions dissuasives lorsque l'interessé cherchait à se déclarer polonais ${ }^{23}$. L'entretien devait se conclure par la signature du formulaire de demande, acte symbolique d'acquiescement qui en fait suscita de nombreuses oppositions ${ }^{24}$. Celles-ci furent massives à Lvov, principal bastion polonais de la région, où les résistances se manifestèrent aussi par des vols massifs de formulaires

19. A. Głowacki, Sowieci...., op. cit., p. 80 .

20. J. T. Gross, Revolution..., op. cit., p. 188-189.

21. Cette estimation, présentée dans l'étude pionnière de K. Kersten, d'après les travaux de A. Kulischer, est depuis systématiquement reprise. K. Kersten, Repatriacja ludności polskiej po II Wojnie Ś wiatowej, Varsovie, Ossolineum (PAN), 1974, p. 31.

22. Ibid. ; A. Głowacki, Sowieci... op. cit., p. 80-81.

23. Voir notamment K. R. Jolluck, Exile and identity. Polish women in the Soviet Union during World War II, Pittsburgh, Pittsburgh University Press, 2002, p. 204-205.

24. J. T. Gross, Revolution..., op. cit., p. 190. 
dans les bureaux d'enregistrement. Sur 40000 passeports prévus, seuls 1500 avaient été délivrés fin mars et la presse officielle locale reconnut elle-même que la campagne avait été mal préparée ${ }^{25}$. Parmi les plus réfractaires à la signature figurèrent certes de nombreux Polonais pour lesquels l'acte signifiait une négation de leur patrie, mais également de nombreux réfugiés qui redoutaient de ne plus pouvoir ainsi retourner dans leurs foyers, au-delà du Bug. L'infléchissement du climat social et politique de l'hiver 1940 s'exprima de façon extrême dans le comportement d'une partie significative des juifs qui, après avoir fui l'occupation nazie, commença à vouloir figurer sur les listes des volontaires au transfert dans le Gouvernement général, ce qui entraîna, comme nous le verrons par la suite, leur déportation ${ }^{26}$.

L'étendue temporelle de la campagne de passeportisation reste jusqu'à présent difficile à préciser, de même que son incidence directe sur les arrestations qui, de fait, s'intensifièrent à partir de février et furent particulièrement massives vers juinjuillet, puis en septembre (plus de 15000 arrestations mensuelles) ${ }^{27}$. Dans cette période, l'attention des autorités centrales se porta davantage sur les républiques baltes, devenues républiques de l'URSS au cours de l'été, tandis que les autorités locales d'Ukraine et de Biélorussie engagèrent à l'automne une campagne de vérification des passeports dans les régions orientales de ces républiques. Ce contexte explique sans doute la relative accalmie dont bénéficièrent les anciens territoires polonais dans la dernière partie de l'année, après la vaste opération d'épuration engagée non seulement par le biais de la passeportisation mais aussi, de façon plus explicite, à travers les déportations de masse.

\section{Les vagues de déportation}

Les liens entre les formes de résistance ou d'exclusion manifestées au cours de la passeportisation et les opérations de déportations réalisées dans la même période n'apparaissent pas formellement à travers les catégories d'individus visées par ces dernières, sinon tardivement lors de la dernière vague de déportation de juin 1941 qui mentionnait, parmi les réprimés, les individus ayant refusé de prendre le passeport soviétique. Néanmoins, le déroulement parallèle de ces deux actions conduit à formuler l'hypothèse d'une forte interaction, à l'exception cependant du premier transfert massif qui intervint en février 1940 et qui fut, contrairement aux autres vagues, exclusivement conçu à l'échelle des pouvoirs centraux.

Le scénario des quatre grandes opérations qui jalonnèrent la période n'a pas attendu l'ouverture des archives soviétiques pour être assez précisément retracé, jusque dans les catégories de groupes socio-professionnels et politiques destinés à

25. S. G. Filippov, « Dejatel'nost'... », art. cit., p. 57; A. Głowacki, Sowieci..., op. cit., p. 8283.

26. J. T. Gross, Revolution..., op. cit., p. 190-192.

27. O. A. Gorlanov, A. V. Roginskij, « Ob arestah v zapadnyh oblastjah Ukrainy i Belorussii v 1939-1941 » (Les arrestations dans les régions occidentales d'Ukraine et de Biélorussie en 1939-1941), A. E. Gur’janov, éd., Repressii protiv Poljakov..., op. cit., p. 88. 
être déportés. Les matériaux collectés et conservés par le gouvernement polonais en exil ont permis de reconstituer l'essentiel de la trame événementielle et ont livré, en outre, de très nombreux témoignages sur le vécu de la déportation ${ }^{28}$. En revanche, d'importants rectificatifs ont été apportés, suite à la consultation des documents du NKVD, aux estimations quantitatives faites par les autorités polonaises et reprises jusqu'à la fin des années 1980. Après avoir longtemps évalué à 1,5 million le nombre d'anciens citoyens polonais déportés, les historiens l'estiment généralement à 320000 environ $^{29}$.

La première opération (en février 1940) et - numériquement parlant - la plus importante fut planifiée sur la proposition de Berija, soumise le 2 décembre 1939 à Stalin. Elle programmait de déporter en priorité les «osadniki », soit ces anciens membres de l'armée qui avaient participé à la guerre polono-russe de 1920 et auxquels furent attribuées des terres, avec l'objectif stratégique d'affirmer la présence polonaise dans ces régions frontalières. Ennemis auto-désignés des Russes, koulaks par définition et privilégiés de l'État polonais, les osadniki représentaient dans l'esprit des autorités soviétiques un groupe socio-politique irréductiblement étranger et néfaste. Les décisions du Politbjuro du CC du PCUS et les décrets du Sovnarkom de décembre 1939 donnèrent lieu, le 10 février 1940, à la déportation de 140000 personnes environ, dont une grande partie vers Arkhangelsk. Les déportés spéciaux de février 1940 étaient, en réalité, en nombre beaucoup plus important que les 6 à 8000 familles d'osadniki installées dans les confins. Ils comprenaient plus généralement des exploitants agricoles et de nombreux employés de l'ancienne administration forestière qui furent ainsi transférés au fin fond de l'URSS avant la mise en œuvre de la campagne de passeportisation.

Les deux vagues de déportations d'avril et de juin furent planifiées au niveau central, avec également la collaboration de Nikita Hruščev, alors premier secrétaire du parti communiste d'Ukraine. Les déportations par voie administrative d'avril concernèrent surtout les représentants de l'ancien ordre public polonais : policiers, gendarmes, gardiens de prison, employés administratifs. Elles visaient aussi les membres des classes possédantes : propriétaires, artisans, fabricants ; les membres d'organisations contre-révolutionnaires ainsi que les familles des individus réprimés. La décision prise le 2 mars relativement à cette deuxième vague mentionnait significativement les familles des officiers dont la mise à mort fut décidée, elle, trois jours plus tard, témoignant, s'il en était besoin, du haut degré de coordination

28. C'est sur la base de cette collection de récits effectués auprès de ceux des Polonais qui furent évacués en 1942 avec l'armée Anders que Jan Gross a reconstitué dans les années 1980 l'histoire des déportations. Voir J. Gross, Révolution..., op. cit.

29. Cette estimation est issue de la recherche de A. E. Gur' janov, « Pol'skie specpereselency v SSSR v 1940-1941 gg. » (Les sepcpereselency polonais en URSS en 1940-1941) et « Masštaby deportacii naselenija v glub' SSSR v mae-ijune 1941 » (L'ampleur de la déportation de la population à l'intérieur de l'URSS en mai-juin 1941), in A. E. Gur'janov, ed., Repressii..., op. cit., p. 114-175. Dans l'exposé qui suit, nous nous référons à cette étude. Bien que les résultats présentés par A. E. Gur'janov soient accrédités par la plupart des spécialistes, certains historiens persistent néanmoins dans de très hautes estimations. Voir M. Tuzsynski, « Soviet war crimes against Poland during the Second World War and its aftermath: a review of the factual record and outstanding questions », Polish Review, 2, 1999, p. 183-216. 
des actions d'épuration, déplacements forcés d'une part, pure éradication de l'autre. Les convois du 13 avril, dirigés surtout vers le Kazakhstan, comprenaient environ 61000 personnes.

La troisième vague de juin, dite des réfugiés, comportait en effet une catégorie spécifique de personnes qui s'étaient fait enregistrer sur les listes de la Commission soviéto-allemande de transferts mais qui n'avaient pas été sélectionnées par les responsables allemands de la commission lorsque ceux-ci s'étaient rendus en Ukraine en mai 1940. Parmi les 75000 personnes qui furent alors déportées vers les colonies spéciales des régions du Nord, figuraient plus de $80 \%$ de juifs, proportion comparable à celle des Polonais dans la première vague de déportations ${ }^{30}$.

Alors que les grandes opérations de masse de l'année 1940 avaient été réalisées selon les décisions du Politbjuro du CC du PCUS et du Sovnarkom, les déportations de mai-juin 1941 ne portent pas trace de telles résolutions, si ce n'est celle du 14 mai 1941, formulée de manière plus imprécise et relative à l'arrestation et au transfert des familles des membres d'organisations contre-révolutionnaires ukrainiennes et polonaises. D'autre part, elles se distinguent des précédentes par leur étendue géographique et temporelle : elles affectèrent tous les nouveaux territoires incorporés, de la Bessarabie à l'Estonie, et se déroulèrent entre mai et juin selon les régions. En Lituanie, l'invasion allemande surprit des convois sur le point de partir et qui furent interceptés. De fait, cette dernière série de déportations s'est en partie confondue avec les grands mouvements d'évacuation entrepris lors de l'arrivée des troupes allemandes. Sur près de 90000 personnes alors déportées vers l'intérieur de l'URSS, le nombre d'anciens citoyens polonais reste difficile à préciser car les statistiques des déportations de Lituanie, où ils formèrent une partie notable des contingents, ne les ont pas mentionnés en tant que tels. Ils auraient constitué, selon les estimations, plus du tiers des déportés (de 34000 à 41000 personnes).

La très grande diversité des destinations, l'inégale durée du vécu dans les colonies spéciales et les camps ne permettent pas d'évoquer, même synthétiquement, ce pan d'histoire qui s'acheva à partir d'août 1941, dans le nouveau contexte de la « grande guerre patriotique ». À cette date près de 390000 anciens citoyens polonais, déportés mais aussi prisonniers de guerre et détenus dans les camps du Goulag, se trouvaient en URSS, selon les rapports ultérieurs du NKVD et le bilan que présenta Vyšinskij au nouvel ambassadeur polonais ${ }^{31}$.

30. Dans le cadre de l'accord germano-soviétique sur les transferts de population, environ 66000 personnes des territoires annexés en 1939 purent partir ou repartir vers la Pologne. M. Wieliczko, « Migracje przez "linię demarkacyjną" w latach 1939-1940 », in M. Marszałka, ed., Potożenie ludności polskiej na terytorium ZSRR i wschodnich ziemiach II Rzeczypospolitej w czasie II Wojny światowej, Toruń, 1990, p. 137.

31. Vyšinskij indiqua à l'ambassadeur de Pologne, S. Kot, la présence de 387932 anciens citoyens polonais présents en URSS (K. Kersten, Repatriacja..., op. cit., p. 34) ; le premier bilan général de Berija mentionnait 389382 personnes (GARF, f. 9401, op. 2, d. $64,1.384-3)$. 


\section{Qu'est-ce qu'un citoyen polonais? \\ La controverse polono-soviétique sur le statut des déportés amnistiés}

La mise en œuvre du plan Barbarossa, qui déclencha la «grande guerre patriotique » en juin 1941, modifia radicalement l'attitude de l'URSS face au gouvernement polonais en exil, partenaire protégé du camp allié que le gouvernement soviétique s'empressa de rejoindre dès les premières semaines de l'agression. Cette nouvelle période de coopération bilatérale, qui se traduisit par le rétablissement de l'ambassade polonaise sur le territoire soviétique et l'arrivée d'une délégation en provenance de Londres, dura près de deux ans et fut tout à fait insolite dans l'histoire soviétique. Jamais, en effet, aucun État étranger ne bénéficia de prérogatives aussi étendues sur le territoire soviétique que celles qui furent concédées au gouvernement polonais dans la prise en charge de ses ressortissants. Cela étant, les concessions faites furent caractérisées par un écart sans cesse croissant entre les accords de principe et les marges d'action réelles des représentants polonais, continuellement enfreintes par les mesures de contrôle et de restriction imposées par le pouvoir central soviétique. De fait, le contentieux latent sur la délimitation des frontières entre les deux États se reporta, compte tenu de l'occupation par les Allemands des territoires en litige, sur les populations qui en avaient été déportées.

Le nouveau cadre de coopération défini par les accords bilatéraux de juillet et août 1941 s'appliquait essentiellement aux ressortissants polonais déportés en 1940-1941. Le principe de leur libération des camps et colonies spéciales dans lesquels ils étaient jusque-là détenus, de la formation en URSS d'une armée polonaise destinée - au terme de laborieuses négociations - à être évacuée via l'Iran, et de la mise en place d'une aide humanitaire aux personnes libérées, conduisit l'ambassade polonaise à mettre en place un vaste réseau de représentants et de responsables, chargés d'enregistrer, d'orienter, de subvenir aux besoins des milliers d'individus et de familles dont le sort nouveau demeurait néanmoins incertain.

La première étape dans la réalisation des accords consistait donc dans la libération des déportés qui fut effectivement proclamée le 12 août 1941 par un ukaz du Présidium du Soviet suprême de l'URSS, lequel ordonnait, dans une formulation particulièrement brève mais impérative, $\mathrm{d}^{\prime}$ ' amnistier tous les citoyens polonais se trouvant actuellement sur le territoire soviétique soit en qualité de prisonniers de guerre, soit sur d'autres fondements $»^{32}$. Les bénéficiaires recevaient à leur sortie une attestation (udostoverenie) qui leur permettait désormais de circuler librement en URSS, à l'exception de certaines zones spéciales ou interdites ${ }^{33}$. Les autorités locales devaient leur rendre tous les documents et les biens confisqués, pourvoir si nécessaire à l'habillement manquant, et fournir des billets de train. Toutes les personnes libérées disposaient d'un délai de trois mois pour se faire enregistrer auprès de l'ambassade et obtenir auprès des autorités locales des permis de séjour pour étrangers.

32. GARF, f. 7523 , op. 10a, d. 69a, 1. 2.

33. Soit les zones frontalières et interdites, les zones de front, les villes soumises à régime spécial de $1^{\text {re }}$ et $2^{\mathrm{e}}$ catégories. 
L'ukaz du 12 août 1941 fut suivi de plusieurs directives du NKVD central qui en limitèrent immédiatement l'application. D'après l'analyse faite de ces instructions par l'historien Albin Głowacki, les restrictions explicites ou potentielles étaient de deux ordres. Elles concernaient en premier lieu certaines catégories de condamnations. Dans les instructions qu'il adressa aux responsables locaux les 19 et 20 août 1941, Berija excluait d'une libération immédiate les individus condamnés pour activité antisoviétique, pour espionnage au profit de l'Allemagne, et pour participation à l'organisation nationaliste ukrainienne de l'OUN (Organizacija Ukrainskih nacionalistov - Organisation des nationalistes ukrainiens) ${ }^{34}$. Ces restrictions furent complétées par la directive, en date du 19 septembre 1941, du vice-commissaire du NKVD, B. Kobulov qui, tout en élargissant la liste des exclus selon les délits, tel celui de déserteur de l'Armée rouge, apporta également d'autres précisions en matière de preuve identitaire des ayants droit. Il indiquait, notamment, qu'il fallait entendre par bénéficiaires « les citoyens polonais en possession d'un passeport soviétique délivré après le 17 septembre 1939» et qu'en revanche «n'étaient pas concernés par l'amnistie les Polonais qui, au moment de leur délit, n'avaient aucune citoyenneté »35. L'introduction de ces conditions limitait beaucoup en théorie la portée de l'amnistie dans la mesure où - on l'a observé - nombre de déportés n'avaient pas le précieux document, soit parce qu'ils avaient été transférés avant même l'opération de passeportisation, soit parce que la délivrance de ce document leur avait été refusée, soit encore parce qu'ils avaient eux-mêmes refusé de prendre la citoyenneté soviétique. Quel fut le degré d'application de cette exigence de preuve ? Lors du contrôle interne du NKVD central consécutif aux plaintes de l'ambassade sur l'application inégale de l'amnistie, il apparut que certains détenus n'avaient pas été libérés faute de papiers d'identité. Ainsi le responsable du camp d'Užtojžemskij justifia auprès de ses supérieurs le maintien en détention de 45 Polonais «qui ne possédaient aucun document confirmant leur citoyenneté polonaise $»^{36}$. Bien des indices conduisent néanmoins à penser que la directive de Kobulov fut loin d'avoir été suivie à la lettre. Elle fut, en règle générale, comprise dans le sens plus large d'une exigence de preuve quelconque concernant l'ancienne sujétion polonaise car la préoccupation première des autorités soviétiques était de pouvoir distinguer les « citoyens polonais » des citoyens soviétiques de nationalité ethnique polonaise réprimés avant 1939, et dont beaucoup se trouvaient dans les mêmes régions de relégation que les «nouveux venus », notamment au Kazakhstan ${ }^{37}$. Dans la pratique, la conception de la preuve identitaire varia consi-

34. A. Głowacki, « Dla kogo “Amnestia” 1941 roku ? », My Sybiracy, 6, 1995, p. 11.

35. Ibid., p. 13. La directive excluait également les citoyens soviétiques qui avaient trahi la patrie, s'étaient enfuis en Pologne où ils avaient reçu une nouvelle citoyenneté. Il est difficile d'interpréter cette dernière mention, à moins d'envisager des cas de personnes déférées par les autorités allemandes aux Soviétiques.

36. Ibid., p. 16.

37. N. F. Bugaj, L. Berija - I. Stalinu : « Soglasno Vašemu ukazaniju... » (L. Berija à J. Stalin: «Conformément à votre ordre... »), Moscou, AIRO-XX, 1995, p. 8-11. Les répressions engagées dans les régions frontalières de l'URSS au cours des années 1930 touchèrent environ 300000 citoyens soviétiques de nationalité ethnique polonaise, dont 140000 durant la Grande Terreur. Voir S. Ciesielski, W. Materski, A. Paczkowski, Represje..., op. cit. , p. 3-5. 
dérablement selon les interlocuteurs des administrations locales. Alexandre Wat relate qu'en guise de papier demandé, il présenta le seul document qui lui restait, à savoir sa carte du Pen club de Varsovie (Union d'écrivains) qui lui permit effectivement de poursuivre ses démarches ${ }^{38}$. Certains responsables locaux cherchèrent à pallier l'absence de preuve en exigeant des requérants qu'ils figurent dans des listes nominatives de l'ambassade ${ }^{39}$. Une proportion, difficile à préciser, de personnes a été de fait libérée sans avoir pour autant présenté un quelconque document. Courant 1942, l'administration soviétique reconnaissait ainsi l'existence de nombreux cas d'apatrides, c'est-à-dire de «sans-papiers », parmi les amnistiés ${ }^{40}$. De son côté, l'ambassade fit part, début 1942, de différentes plaintes des personnes libérées concernant la confiscation, lors des enregistrements à la milice, des attestations d'amnistie qui constituaient les seuls documents en leur possession ${ }^{41}$.

Ces certificats représentaient, dans l'histoire de la contrainte documentaire subie depuis 1939 par les déportés, le premier document positif, la manifestation tangible de la réparation d'une injustice, le symbole de la liberté. Il est significatif, de ce point de vue, que de nombreux enfants, relatant cette nouvelle période dans leur récit de déportation, aient mentionné cette étape notoire que fut la délivrance des attestations d'amnistie, témoignant également par là de leur pleine intériorisation du rôle crucial des documents pour tout résident sur le territoire soviétique ${ }^{42}$. «Quand j'ai reçu mes documents - écrivit un adolescent - je suis redevenu un homme libre après deux ans. » ${ }^{43}$ Après avoir été l'objet d'une stigmatisation réelle ou ressentie telle, la qualité de Polonais ouvrait désormais la porte des camps et des colonies spéciales, constituait la promesse d'un avenir plus heureux et manifestait enfin l'existence - à défaut de pays — d'une réalité nationale qui semblait jusquelà anéantie. Cette radicale inversion de statut était évidemment propice au développement massif d'un sentiment identitaire polonais parmi les déportés, fussent-ils juifs, biélorusses ou ukrainiens ${ }^{44}$.

Désormais les anciens déportés furent assimilés à une nouvelle catégorie de population en pleine croissance qui était celle des «évacués ». La libération des citoyens polonais coïncida en effet avec la période des grandes migrations internes de Soviétiques vers l'est, qui avaient commencé au début de l'été et s'étaient inten-

38. A. Wat, Mon siècle. Confession d'un intellectuel européen, Paris, L'Âge d'homme, 1989, p. 653 .

39. E. Raczyński, B. Biegański, eds, Documents on Polish-Soviet relations 1939-1945, t. I, 1939-1943, Londres - Melbourne - Toronto, Heinemann, 1961, p. 230 (cité infra: Documents...).

40. GARF, f. 9415 , op. 3, d. $1400,1.4$.

41. Documents..., op. cit., p. 323, 343 .

42. J. T. Gross, I Grudzinska, eds, War through children's eyes. The Soviet occupation of Poland and the deportations, 1939-1941, Stanford, HIP, 1981, p. 84, 89, 107, 111, 130, 158, 230,236 . De nombreux récits d'enfants ne vont pas cependant jusqu'à l'amnistie et décrivent essentiellement l'histoire de leur déportation.

43. Ibid, p. 220.

44. K. R. Jolluck, Exile and identity..., op. cit., p. 199-212. 
sifiées lors de l'attaque allemande en direction de Moscou. Plus de 17 millions de personnes ont été ainsi évacuées des zones occidentales au cours de la guerre ${ }^{45}$. L'ambassade polonaise elle-même partit, à l'instar de la majorité des institutions soviétiques et étrangères, à Kouïbychev en octobre 1941 et ce transfert contraria fortement son activité. La rigueur théorique des instructions du NKVD ne pouvait masquer l'état de débâcle dans lequel se trouvait alors le pays. Quelle en fut l'incidence sur la conduite des amnisties ?

Le degré d'application de l'ukaz d'amnistie peut être apprécié diversement selon les sources et les dires des témoins. Ces derniers mentionnent le plus souvent le caractère tardif des libérations, et les limites imposées à la liberté de circulation ${ }^{46}$. Beaucoup d'anciens déportés ont également rapporté que nombre de Polonais n'avaient pas été amnistiés. Il est néanmoins difficile de savoir dans quelle mesure ceux-ci étaient bien des « anciens citoyens polonais » et non des Polonais soviétiques. De son côté, l'ambassade a régulièrement dénoncé les maintiens abusifs en détention, mais, tout en s'appuyant sur des cas bien identifiés de rétention, elle n'avait pas de critères d'évaluation fiables de la population déportée des territoires annexés et l'avait beaucoup surestimée. D'autre part, l'inquiétude des représentants polonais concernant l'application de l'amnistie était aussi motivée par l'absence des 15000 officiers prisonniers de guerre sur le sort desquels ils ne cessèrent d'interroger les autorités soviétiques jusqu'à la découverte des charniers de Katyń. Dans les faits, le département de l'ambassade, qui s'était spécifiquement consacré au suivi de l'amnistie, intercéda sans relâche en faveur de la libération de ses ressortissants sur la base de requêtes personnelles, et il envoya, durant les 21 mois de coopération soviéto-polonaise, 79 notes au NKID concernant 5579 condamnés et 1348 colons spéciaux ${ }^{47}$.

Si l'on se réfère aux sources soviétiques sur le bilan de l'amnistie établi au $1^{\text {er }}$ septembre 1942 et régulièrement rappelé dans les rapports postérieurs du NKVD sur les «anciens citoyens polonais », seules 341 personnes restaient en détention à cette date ${ }^{48}$.

45. R. Manley, «L'URSS en guerre : la question de l'évacuation de la population civile », Communisme, 70-71, 2002,p. 159-179.

46. S. Ciesielski, Polacy w Kazachstanie w latach 1940-1946, Wrocław, 1997, p. 65 sq. ; J. T. Gross, I. Grudzińska-Gross, W czterdziestym nas matko na Sybir zesłali. Polska a Rosja 1939-1942, Varsovie, 1990, p. 267 ; P. Žaron, «Deportacija pol'skogo naselenija v Sibir' (1940-1941) i repatriacija na rodinu (1945-1949) » (La déportation de la population polonaise en Sibérie (1940-1941) et son rapatriement dans la patrie (1945-1949)), in A. Kučinskij, P. Romanov, Sibir' v istorii i kul'ture pol'skogo naroda (La Sibérie dans l'histoire et dans la culture du peuple polonais), Moscou, Ladomir, 2002, p. 382-384.

47. K. Sword, Deportation and exile. Poles in the Soviet Union, 1939-1948, Londres, Macmillan Press, 1994, p. 52-53. Le bureau reçut des réponses concernant 3229 cas, mais on ne sait pas quels furent les résultats définitifs de ces démarches.

48. GARF, f. 9401 , op. 2, d. 64,1.384. 


\section{L'imposition d'une « citoyenneté ethnique »}

Bien qu'ayant été dans nombre de cas très contraignante, la nécessité de la preuve documentaire s'avéra être un moindre mal par rapport aux restrictions introduites par la suite. Lorsque le bénéficiaire avait passé ce premier obstacle administratif, il recevait alors une attestation indiquant, à la suite de ses nom et prénom, que le porteur « [était] amnistié comme citoyen polonais sur les fondements de l'ukaz du Soviet suprême et qu'il a[vait] le droit de circuler librement sur le territoire de l'URSS »49. Mais l'évidence, dans la formulation, de la définition du «citoyen polonais » fut singulièrement remise en cause lorsqu'il s'agit effectivement de procéder à la régularisation des ex-déportés par la délivrance d'un passeport national.

C'est une note du commissaire du peuple à la Guerre du Kazakhstan qui alerta, début novembre 1941, les autorités polonaises sur leurs prérogatives concernant les ressortissants polonais. À la suite de plaintes émises par l'ambassade sur l'incorporation forcée de citoyens polonais dans l'Armée rouge, le commissaire avait tenu à préciser qu'il agissait conformément aux directives du gouvernement central, lesquelles avaient indiqué de considérer comme citoyens soviétiques tous les anciens citoyens de la république de Pologne ayant une autre origine que polonaise et possédant un passeport soviétique ${ }^{50}$. Interrogé à ce sujet par les autorités polonaises, le commissariat du peuple aux Affaires étrangères énonça officiellement la position soviétique en matière de citoyenneté dans sa note du $1^{\text {er }}$ décembre, soit à une date correspondant peu ou prou à l'expiration des délais d'enregistrement impartis aux premiers bénéficiaires de l'amnistie. Dans cette note, il était rappelé que

en vertu du décret du 29 novembre 1939 tous les citoyens des républiques d'Ukraine et de Biélorussie occidentales ont acquis la citoyenneté soviétique et que si le gouvernement soviétique est prêt à reconnaître la citoyenneté polonaise aux personnes de nationalité (ethnique) polonaise, ce fait témoigne surtout de sa bonne volonté et de sa tempérance. Il ne faut en aucun cas en faire le fondement d'une reconnaissance comme citoyens polonais des individus d'autres nationalités, en particulier ukrainienne, biélorusse et juive ${ }^{51}$.

Une nouvelle étape était désormais franchie sur le seuil de l'éligibilité. Il ne suffisait plus de prouver son ancienne qualité de citoyen polonais, il fallait désormais démontrer sa « polonité » ethnique pour bénéficier de la protection de l'ambassade et plus concrètement pour espérer, dans un terme toujours imprécis, quitter l'URSS. L'intrusion du critère ethnique dans la conception soviétique de la citoyenneté polonaise annonçait clairement le franchissement d'une nouvelle étape dans la relation bilatérale par la réactualisation du différend sur le tracé des frontières, il conte-

49. S. Ciesielski, Polacy..., op. cit, p. 64 (fac-similé du formulaire d'attestation).

50. Documents..., op. cit., p. 200.

51. Documents..., op. cit., p. 227; GARF, f. 9415, op. 3, d. 1400,1. 2. 
nait, en effet, tout l'argumentaire jusqu'alors déployé pour justifier l'annexion des territoires en litige, à savoir que les Polonais ethniques y avaient été minoritaires et ne pouvaient donc prétendre à une quelconque légitimité en tant que nation dominante et qu'en revanche l'existence de républiques ukrainienne et biélorusse justifiait le rattachement à ces États de régions peuplées en majorité par des populations de ces nationalités.

L'introduction de la condition ethnique dans la définition de la citoyenneté polonaise menaçait désormais la pleine réalisation de l'ukaz d'amnistie, alors que les instructions concernant son application n'avaient jusque-là comporté aucune clause relative à la nationalité.

S'agissant, en revanche, du processus de régularisation des documents d'identité des amnistiés, les archives centrales témoignent de la précocité de l'adoption de la « position soviétique » en matière d'attribution de la citoyenneté. L'absence, à ce jour, de trace de directives spécifiques sur le sujet est en partie compensée par les correspondances administratives internes, tel ce courrier de demande de précision du 2 octobre 1941 adressé par Serov, vice-commissaire du NKVD à Vyšinskij, qui illustre déjà à cette date la pleine intégration par l'administration du NKVD des critères ethniques à appliquer pour l'attribution de la citoyenneté polonaise, la question posée portant, elle, sur la procédure de « dénaturalisation » :

Parmi les citoyens polonais libérés par l'amnistie [...] ayant l'ancienne citoyenneté polonaise et étant [....] citoyens de l'URSS, ceux qui ont reçu à l'ambassade polonaise des passeports nationaux ont demandé à ce qu'on leur délivre des papiers en tant qu'étrangers. En relation avec ce fait, je vous demande de préciser : doit-on reconnaître comme citoyens polonais les individus de nationalité polonaise présentant des passeports polonais mais étant des citoyens soviétiques ou bien la «sortie» de la citoyenneté soviétique doit-elle être effectuée conformément à la loi soviétique du 19 août 1938 ?52

Dès le début de la nouvelle opération d'enregistrement, le NKVD possédait donc une bonne maîtrise des consignes passées. Celles-ci allaient encore être complétées dans un sens plus restrictif par Vyšinskij au cours des six premiers mois de l'année 1942.

Cette période correspond au moment d'activité le plus intense des autorités polonaises sur le territoire soviétique, l'année s'ouvrant sur la création d'une vingtaine de représentations consulaires, l'organisatisation de transferts des premières unités constituées dans la région de la Volga vers les républiques d'Asie centrale, Kazakhstan et Ouzbekistan principalement, où furent répartis en différents camps les membres de l'armée Anders en formation, l'État-Major polonais s'étant, lui, localisé à Yangi-Yul près de Tashkent. La présence de ces centres, qui comptaient

52. GARF, f. 9415, op. 3, d. 1400, 1. 1. L'auteur se référait à l'article 4 de cette loi relatif à l'examen des demandes de sortie de citoyenneté par le Présidium du Soviet suprême de l'URSS, ce qui supposait que tous les dossiers des bénéficiaires fissent l'objet d'une procédure de contrôle supplémentaire au niveau central et non plus seulement à l'échelle des organes du NKVD. 
environ 64000 hommes à la mi-mars 1942, attira également de très nombreux civils, familles des militaires bien souvent, mais pas exclusivement, qui arrivèrent de tous les points de l'URSS. Un véritable exode interne s'était esquissé au cours de l'hiver 1941-1942 vers les républiques d'Asie centrale qui allait aboutir, pour près de 120000 personnes, au départ de l'URSS. L'organisation en deux temps des évacuations du printemps et de la fin de l'été 1942, sur fond d'âpres négociations bilatérales, tant sur le principe même des départs que sur leur destination et leur logistique, fut accompagnée, côté soviétique, par de nouvelles instructions en matière de contrôle des identités.

Le 30 mars 1942, Vyšinskij adressa un courrier au vice-commissaire du NKVD pour apporter de nouvelles corrections aux critères de reconnaissance des citoyens polonais. Il fit savoir en effet que les ayants droit de nationalité ethnique polonaise ne concernaient que les individus originaires d'Ukraine et de Biélorussie occidentales.

Les personnes de nationalité polonaise arrivées pour vivre en Lituanie, Lettonie, Estonie après le 17 septembre $1939^{53}$, ayant acquis là-bas la citoyenneté de l'un de ces États et étant devenus citoyens de l'URSS, ne [devaient] pas être considérées comme citoyens polonais ${ }^{54}$.

L'introduction de cette nouvelle clause restrictive visait principalement les Polonais de Wilno et de ses proches alentours, ce territoire que les Lituaniens avaient vainement revendiqué, avec le soutien de la SDN aux lendemains de la Première Guerre mondiale et qui leur avait été restitué en octobre 1939 par les Soviétiques, au cours d'une brève intervention. Ceux-ci avaient laissé les Lituaniens prendre pleinement possession de leur nouvelle capitale avant d'engager en juin 1940 l'offensive qui allait conduire à l'incorporation de la république à l'URSS. Dans l'intervalle, le gouvernement lituanien exerça de très fortes pressions pour soumettre les Polonais locaux à la nouvelle réalité étatique-nationale, notamment par la promulgation, en décembre 1941, d'une loi qui conditionnait l'exercice de nombreuses professions et fonctions à l'adoption de la citoyenneté. Cette loi applicable à toute personne résidant dans la république le 27 octobre 1939 touchait non seulement la population permanente mais également les groupes de réfugiés en provenance de Pologne centrale dont le nombre était alors estimé à $30000^{55}$. Prétextant de l'« effacement » de la sujétion polonaise par l'acquisition de la citoyenneté d'un État balte antérieurement à la soviétisation des républiques, le vice-commissaire du NKID donna, en quelque sorte, le signal d'un nouveau contrôle.

La directive de Vyšinskij intervint au beau milieu de la première évacuation qui se déroula du 25 mars au 5 avril 1942 et comprit au total près de 44000 personnes,

53. Dans la note de mai 1942 qu'il adressa au vice-commissaire Merkulov, Vyšinskij recommandait de supprimer le renvoi au 17 septembre 1939 et de considérer comme Soviétiques tous les Polonais ayant pris la citoyenneté de l'une des républiques baltes.

54. GARF, f. 9415 , op. 3, d. 1400,1.3.

55. P. Łossowski, Litwa a sprawy polskie 1939-1940, Varsovie, Nowa, 1985, p. 112-116.

L. Tomaszewski, Kronika Wileńska 1939-1941, Varsovie, Pomost, 1990, p. 34-36. 
selon les données d'enregistrement au port d'arrivée à Palhevi ${ }^{56}$. Sans pouvoir apprécier le rôle de cet appel au redoublement de vigilance et de sélection dans la conduite des opérations d'évacuation, son incidence dans les communautés polonaises en URSS fut attestée à travers la réaction rapide du gouvernement polonais à Londres qui, dès le 13 avril, dénonça auprès de l'ambassadeur de l'URSS, Bogomolov, l'incorporation massive des citoyens polonais dans l'Armée rouge, y compris «ceux dont la nationalité polonaise est expressément reconnue dans la note du NKID du $1^{\text {er }}$ décembre $1941 »^{57}$.

Le durcissement du contrôle soviétique sur les identités fut confirmé en mai 1942 par une nouvelle note du NKID au NKVD relative à un projet d'instruction sur la documentation et l'enregistrement des Polonais. La nouvelle réglementation introduite en matière de délivrance des passeports revenait à supprimer toute prérogative aux autorités polonaises, qui étaient désormais sommées de présenter des listes nominatives, en quatre exemplaires, de tous ses requérants, le NKID disposant du pouvoir décisionnel dans la reconnaissance ou non de la citoyenneté polonaise ${ }^{58}$. Vyšinskij répondait ainsi tardivement à la requête adressée par Serov au tout début des opérations d'enregistrement, en introduisant effectivement une procédure de contrôle au niveau central, exercée non pas par le Soviet suprême mais par le NKID. Tout en confirmant l'exclusion, dans les ayants droit, des Polonais «baltes », l'instruction contenait néanmoins une ouverture qui allait se révéler importante par la suite et qui concernait les résidents temporaires. La note précisait à leur propos que les permis de séjour pour étrangers étaient délivrés « aux citoyens polonais, indépendamment de leur nationalité, lorsqu'ils étaient arrivés en provenance du dit "Gouvernement général" sur le territoire de l'URSS après le 1er novembre 1939 et qu'ils n'avaient pas accompli les formalités légales d'acquisition de la citoyenneté soviétique ${ }^{59}$. Mais encore fallait-il pouvoir prouver la date de son arrivée...

L'introduction de la nouvelle procédure imposée à l'ambassade, qui allait tant susciter l'indignation des autorités polonaises, conduit à explorer plus concrètement les critères sur lesquels les Soviétiques s'appuyaient pour statuer sur la nationalité ethnique des individus. Le formulaire des listes nominatives qui devaient être soumises par l'ambassade au NKID contenait, en effet, 15 paragraphes qui, outre les données courantes de l'état civil, les précisions à fournir sur les trajectoires ${ }^{60}$, exigeaient également un certain nombre d'informations sur les parents (nom, citoyenneté ancienne et actuelle, lieu de résidence passé et présent). Le formulaire comprenait d'autre part un paragraphe sur la nationalité et sur la religion des requérants. En revanche, il ne comportait aucune question sur la langue maternelle qui

56. K. Sword, Deportation..., op. cit., p. 71. Cette première évacuation comprenait près de 11000 civils.

57. Documents..., op. cit., p. 322-323

58. GARF, f. 9415 , op. 3, d. $1400,1.4$

59. Ibid.

60. Localisation jusqu'en novembre 1939, date éventuelle d'arrivée sur le territoire soviétique, date et lieu de délivrance du certificat d'amnistie, lieu de résidence lors du dépôt de la demande d'enregistrement. 
représentait pourtant jusque-là une composante importante de la conception soviétique de l'identité nationale ${ }^{61}$. Celle-ci, dans le cas présent, empruntait essentiellement les critères généalogiques et culturels (à travers la religion principalement) qui caractérisaient jusqu'alors l'appréhension polonaise de l'appartenance ethnique... Compte tenu du nombre d'informations demandées, les pratiques de distinction de la nationalité ethnique paraissaient reposer sur un « faisceau » d'indices probants. Mais si l'on se réfère à l'opération de passeportisation de 1943, la fixation de la nationalité semble surtout avoir été effectuée par référence au nom des personnes, voire à ceux de leurs parents ${ }^{62}$.

Les formes de contrôle de l'identité peuvent très inégalement être reconstituées selon les situations, les enregistrements lors des évacuations vers l'Iran étant les mieux connus, quoique leur degré de contrôle ait, en réalité, largement dépendu des interlocuteurs locaux ${ }^{63}$. Près de $8 \%$ de l'ensemble des personnes transférées dans le cadre du départ de l'armée Anders furent, selon les données polonaises, des membres de minorités juive (3\%), biélorusse $(2,75 \%)$, ukrainienne $(1 \%)$ et autre $(1 \%)^{64}$. Le durcissement du contrôle soviétique s'est surtout exprimé dans le cadre de la deuxième évacuation qui se déroula du 9 août au 1er septembre 1942 et qui donna lieu, lors de sa préparation, à des protocoles visant à éviter que des « citoyens soviétiques » ne partent avec les Polonais ${ }^{65}$. Certains transferts furent négociés, tel celui de ces dix enfants juifs d'un orphelinat de Kermine que relate l'un d'entre eux en rappelant que ses deux frères, eux, durent rester avec les 90 autres enfants juifs non inscrits sur les listes de partants ${ }^{66}$. Qu'il s'agisse de militaires ou de civils, les «cas négociés » semblent avoir concerné essentiellement des juifs, les informations livrées par les sources polonaises étant pratiquement silencieuses sur la présence des Biélorusses et des Ukrainiens ${ }^{67}$.

61. J. Cadiot, « La constitution des catégories nationales dans l'Empire de Russie et en Union des républiques socialistes soviétiques (1897-1939) : statisticiens, ethnographes et administrateurs face à la diversité du "national" », thèse de doctorat, Paris, EHESS, 2001.

62. «Paszportyzacja », Karta, 10, 1993,p. 130.

63. K. Sword, Deportation..., op. cit., p. 56. La commission initiale de recrutement polonosoviétique qui allait par la suite servir de référence à toute les nouvelles commissions locales d'enregistrement pour les évacuations comprenait un représentant militaire polonais, un représentant militaire soviétique et un responsable du NKVD. S'agissant des modalités de contrôle, A. Wat rappelle par exemple qu'il lui fut proposé de partir avec l'armée, bien que juif, en échange d'une bouteille de vodka. A. Wat, Mon siècle..., op. cit., p. 649

64. K. Sword, Deportation..., op. cit., p. 59.

65. Ibid, p. 78-79. Les civils pouvaient prétendre au départ s'ils étaient membres des familles de militaires, fait qui devait être attesté par les autorités militaires polonaises. L'été 1942 marqua à l'évidence une nouvelle étape dans la détérioration des relations polono-soviétiques, qui s'est traduite en juillet par plusieurs arrestations de représentants de l'ambassade, lesquelles coïncidaient, précisément, avec le temps fort de l'activité d'enregistrement pour désigner les partants ; voir, à ce propos, Documents..., op. cit., p. 401 sq.

66. J. Gross, I. Grudzińska-Gross, W czterdziestym, op. cit., p. 230-234.

67. Constat qui est rappelé dans la majeure partie des travaux reposant sur les sources polonaises. Voir notamment C. Jolluck, Exile and identity..., op. cit; K. Sword, Deportation and exile..., op. cit. 
Le contrôle au départ n'a guère concerné plus d'un tiers de l'ensemble des anciens citoyens polonais et la contrainte administrative exercée à l'encontre des centaines de milliers restants, dispersés en groupes très inégaux sur l'ensemble du territoire soviétique, s'est principalement exprimée lors de la nouvelle campagne de passeportisation de l'hiver 1943. La situation, jusque-là, des Polonais libérés est d'une extrême diversité. Au Kazakhstan où ils s'étaient le plus massivement regroupés au cours des migrations de l'hiver 1941-1942, les ex-déportés furent rapidement acculés à trouver, faute de moyens de subsistance, un emploi. Beaucoup furent orientés vers des kolkhozes et de fait se redispersèrent, partageant l'existence très précaire de la population locale face à laquelle pourtant ils apparaissaient bien souvent comme des privilégiés, du moins lorsqu'ils possédaient des passeports soviétiques (les kolkhoziens en étant par définition dépourvus), et lorsqu'ils purent bénéficier de l'assistance humanitaire. La tendance naturelle à former des solidarités communautaires fut stimulée, dans cette période, par le développement de l'aide humanitaire, à travers la distribution de produits alimentaires et de première nécessité importés de l'étranger. L'acheminement des vivres, des vêtements, des médicaments donna lieu à la création d'un réseau relativement important d' « hommes de confiance » (męża zaufania), évalués à près de 400 fin 1942 , qui furent généralement nommés par les représentants de l'ambassade au sein des collectivités locales, et qui instauraient eux-mêmes leur propre relai de distribution interne. L'aide matérielle, même symbolique pour certains, était la matérialisation d'une protection pour les bénéficiaires, le signe d'un privilège pour les témoins soviétiques ou d'une exclusion pour ceux des citoyens polonais auxquels elle fut refusée. Par les conflits qu'elle suscita, en particulier entre juifs et Polonais, l'œuvre d'assistance constitue l'un des laboratoires d'analyse des comportements « ethniques », montrant, à l'autre extrême de l'assignation administrative, la réalité des formes de reconnaissance et de discrimination au sein même des groupes d'exdéportés. L'actualité du critère ethnique ne résidait donc pas seulement dans son enjeu politique sur la scène bilatérale, dans son utilisation comme instrument de pression et de contrainte, elle renvoyait aussi aux comportements collectifs des intéressés ${ }^{68}$. On ne peut ici qu'évoquer cette dimension complémentaire des réalités identitaires qui s'exprimèrent dans un mouvement d'interaction, encore difficile à préciser, avec les pratiques édictées d'en haut et conduites par les acteurs locaux.

L'assistance humanitaire, comme le rappela un mémoire ultérieur du gouvernement polonais à Londres, constitua, de fait, l'une des grandes activités des

68. Outre l'étude spécifique de K. Sword qui aborda la question des relations inter-ethniques dans le cadre de l'aide humanitaire («The welfare of Polish Jewish refugees in the USRR, 1941-1943. Relief supplies and their distribution », in N. Davies, A. Polonsky, eds, Jews in Eastern Poland and the USRR, 1939-1946, Londres, 1991, p. 145-160), de nombreux témoignages et éléments d'analyse sur les relations inter-ethniques parmi les déportés ont été recueillis, fournissant des éclairages très divers, qui mettent tous en évidence l'intériorisation forte de l'appartenance ethnique au sein des groupes de déportés, mais ne permettent pas encore d'apprécier l'importance des clivages existants. Voir à ce propos K. R. Jolluck, Exile and identity..., op. cit.; S. Ciesielski, Polacy..., op. cit.; A. E. Gur'janov, « Pol’skie specpereselency...», art. cit. ; J. Siedlecki (T. Piesakowski), Losy Polaków w ZSRR w latach 1939-1986, Varsovie, 1989, J. T. Gross, I. Gross-Grudzińska, W czterdziestym, op. cit. 
représentants polonais après les évacuations de 1942, qui disparut, du moins en tant que prérogative de l'ambassade, lors de la phase critique des tensions polono-sovétiques de janvier $1943^{69}$.

Le tournant marqué par la victoire de Stalingrad correspondit en effet à une crise ouverte dans les relations bilatérales au cours de laquelle s'exprima le contentieux sur les régions annexées. La position offensive de l'URSS sur le plan militaire et la perspective d'une reprise en mains des territoires occupés par l'Allemagne apportaient une acuité nouvelle à la question du devenir des régions frontalières. Cette position se manifesta par la rupture effective des principaux accords de juillet 1941 relatifs aux Polonais en URSS.

\section{Un débat détourné : citoyenneté et résidence}

Le 16 janvier 1943, une note du NKID à l'ambassade fit savoir que le régime d'exception accordé aux individus de nationalité polonaise avait cessé d'exister et que la possibilité de ne pas leur appliquer la citoyenneté soviétique n'était plus valide ${ }^{70}$.

Parallèlement à cette décision, le gouvernement procéda à la confiscation de tous les biens et institutions polonais en URSS qui relevaient jusqu' alors des prérogatives de l'ambassade (décret du Sovnarkom de l'URSS du 15 janvier) et engagea une nouvelle campagne d'attribution des passeports soviétiques à tous les Polonais présents en URSS.

Dans cette phase ultime de confrontations polono-soviétiques sur les fondements de la citoyenneté, la controverse se polarisa non plus sur l'appartenance ethnique des individus, mais sur leur statut de résidents temporaires ou de résidents permanents. A priori, et en admettant comme un fait acquis l'incorporation définitive des territoires annexés à l'URSS, les enjeux du débat renvoyaient en premier lieu à la situation des réfugiés, arrivés dans les nouvelles régions soviétiques à partir de septembre 1939. Lors des différentes rencontres avec l'ambassadeur Romer, Stalin et Molotov se référèrent régulièrement à l'ukaz du 29 novembre 1939 qui, selon eux, distinguait clairement «les résidents permanents des territoires rattachés à l'URSS, qui avaient pris la citoyenneté soviétique par la force de la loi, des résidents temporaires dont l'application était matière à examen individuel $\gg^{71}$. Ce texte, on l'a vu, était loin d'apporter les précisions que revendiquaient les dirigeants soviétiques ${ }^{72}$. Mais d'une certaine manière, les choses s'étaient bien passées comme ils le prétendaient, puisque la campagne de passepor-

69. Documents..., op. cit., p. 476.

70. Ibid., p. 473.

71. Ibid., p. 494-495. En fait, comme nous l'avons vu, ce décret était loin d'être précis quant aux individus concernés par la citoyenneté soviétique.

72. D'ailleurs de façon significative, Molotov qui avait promis de fournir copie du texte à Romer ne la lui fit jamais parvenir. Documents..., op. cit., p. 512. 
tisation de 1940 avait, semble-t-il, beaucoup contribué à l'exclusion des réfugiés en provenance de Pologne centrale, les acculant à fuir les zones passeportisées. La notion de résident temporaire concernait, en principe et avant tout, cette population qui comptait une majorité de juifs, déportés en juin 1940, auxquels avait été dénié, à partir de l'automne 1941, le droit à la citoyenneté polonaise. Dans les faits cependant, la notion de résident temporaire en débat ne faisait référence qu'au personnel direct et délégué de l'ambassade désormais menacé par la nouvelle campagne de passeportisation, comme l'indiquaient clairement les cas concrets évoqués, de part et d'autre, lors des entretiens bilatéraux ${ }^{73}$. C'est pour ces représentants, venus en URSS à partir de l'été 1941, que Vyšinskij avait envisagé la reconnaissance de la citoyenneté polonaise indépendamment de leur nationalité, à la condition - avait-il précisé - que les individus ainsi visés fussent arrivés sur le territoire soviétique après le $1^{\text {er }} 2$ novembre 1939 . Or l'application de cette clause conditionnelle ressortait des propos de l'ambassadeur qui s'indignait que ses subordonnés eussent à fournir une attestation de leur localisation en novembre $1939^{74}$. La tyrannie de la preuve devenait là l'expression la plus achevée de l'arbitraire soviétique.

L'hiver 1943 fut pour les autorités polonaises une période d'impuissance et de renoncement en faveur des ex-déportés. Outre la défense de ses agents, qu'ils cherchaient à préserver de l'assimilation aux populations des territoires annexés, les représentants du gouvernement en exil avaient également de nouvelles causes à plaider. Il est significatif de ce point de vue que le premier entretien entre l'ambassadeur Romer et Stalin, qui ait fait suite à la note de janvier 1943, n'ait pas concerné en premier lieu le destin des anciens ressortissants mais celui des «malgré-nous » polonais, enrôlés de force dans la Wehrmacht et désormais détenus en URSS parmi des centaines de milliers de soldats de l'armée de Von Paulus ${ }^{75}$. La victoire de Stalingrad signait bien l'abandon du combat mené pendant plus d'un an pour la reconnaissance de la sujétion polonaise.

\section{Des citoyens soviétiques confirmés}

La campagne de passeportisation de l'hiver 1943 apparut avant tout comme le marqueur du tournant politique offensif de l'URSS face aux autorités polonaises. Elle ouvrit, après les espoirs fondés sur l'amnistie de 1941, une nouvelle page sombre et à plusieurs égards paradoxale dans l'histoire des «anciens citoyens polonais » déportés qui, en dépit de leur (re)soviétisation forcée, furent maintenus en état de forte extériorité par rapport à l'ensemble de la société soviétique.

La nouvelle opération d'enregistrement, décrétée simultanément par le Sovnarkom de l'URSS et le CC du PCUS le 14 janvier 1943, ne peut qu'être très partiellement abordée à travers les sources soviétiques, non encore déclassifiées, à

73. Ibid., p. 504-510.

74. Ibid., p. 495.

75. Ibid., p. 489 sq. 
l'exception des résultats statistiques de cette action qui rendent compte, notamment, des mesures répressives auxquelles elle donna lieu ${ }^{76}$. Les deux directives du NKVD du 9 février 1943 sur la procédure à suivre marquèrent, semble-t-il, le début de la passeportisation qui s'adressa en priorité à tous ceux qui, de près ou de loin, représentaient les autorités polonaises sur le territoire de l'URSS. Bien que celles-ci eussent dénoncé la nouvelle procédure, les agents du NKVD se targuaient auprès des « anciens citoyens polonais » d'agir avec le plein aval de l'ambassade, restée en fonction jusqu'en avril. Les pressions se concentrèrent en particulier sur les « hommes de confiance » qui, en tant que personnalités-clés au sein des communautés locales, pouvaient par leur soumission forcée faciliter celle du plus grand nombre ${ }^{77}$. La campagne de passeportisation se déroula selon un scénario bien expérimenté de convocations dans les organes locaux de la milice, d'interrogatoires sur les trajectoires, les opinions et affiliations politiques, les liens éventuels avec les « officiels polonais » déjà assimilés, dans l'esprit des agents du NKVD, à des espions. À cet égard, les familles de militaires qui avaient des passeports avec des visas anglais se trouvèrent particulièrement exposées à la vigilance suspicieuse et menaçante des agents. La confiscation des documents présentés était la norme mais l'absence de possession de pièces d'identité constituait un argument de pression supplémentaire, la situation de l'intéressé étant alors jugée illégale ${ }^{78}$. Si le refus de signer le formulaire d'obtention de la citoyenneté soviétique fut alors assimilé à un acte de résistance nationale, les réfractaires furent aussi des juifs et Alexandre Wat ponctue de façon significative son témoignage de la guerre en URSS en distinguant l'avant de l'après de la passeportisation; il rappelle le climat d'oppression qui commençait à régner dans cette période au sein des différentes communautés d' «anciens citoyens polonais $»^{79}$. Comme en 1940 , les documents délivrés étaient hiérarchisés selon le profil socio-politique des individus, allant d'un passeport soviétique d'une validité de cinq ans à des attestations de trois mois renouvelables qui revenaient de fait à des situations de garde à vue. D'après le bilan soviétique de l'opération, 1583 personnes furent explicitement incriminées pour avoir refusé le passeport ${ }^{80}$ et près de 1500 le furent pour d'autres motifs, notamment d'espionnage, au cours de la campagne ${ }^{81}$.

76. Politbjuro CK RKP(b)-VKP(b) 1919-1952, katalog, t. 3, 1919-1952, Moscou, Rosspen, 2001 , p. 285 , d. $1046, \mathrm{n}^{\circ} 155$ «O sovetskom graždanstve nekotoryh kategorij $\mathrm{B}[\mathrm{y} v$ ših]. Pol'skih graždan » (Sur la citoyenneté soviétique de certaines catégories d'ex-citoyens polonais), (papiers spéciaux). Le catalogue concernant les décisions du Sovnarkom de l'année 1943 ne contient, lui, aucune indication de décret sur la passeportisation.

77. « Paszportyzacja », art. cit., p. 118-119. Cet article présente des extraits du rapport effectué par le premier secrétaire d'ambassade, Stefan Gacki, sur la base de 153 documents et récits de passeportisés. Voir également Documents..., op. cit., p. 504-507; 514-516.

78. « Paszportyzacja », art. cit., p. 121-124.

79. A. Wat, Mon siècle..., op. cit. Sur les résitances manifestées parmi la minorité juive, voir K. R. Jolluck, Exile and identity..., op. cit., p. 205 sq. ; « Paszportizacja », art. cit., p. 125.

80. La peine encourue dans ce cas était une détention en prison pour une période de deux ans, en théorie du moins.

81. GARF, f. 9401, op. 2, d. 64, 1. 384-3. Parmi ces 1500 personnes, la majorité (969) était détenue sur la ligne du NKGB. 
Pour les autorités polonaises, la passeportisation fut vécue comme une insupportable provocation, pour les anciens ressortissants elle ne marqua pas seulement la soumission, mais aussi le retour à la répression directe ou potentielle, par les formes de stigmatisation et d'inquisition qu'elle fit renaître. Pour les autorités soviétiques, la passeportisation fut certes un signal politique à l'égard de l' « allié » polonais, un moyen de démanteler les réseaux de l'ambassade sur le territoire soviétique et, plus généralement, un instrument d'épuration efficace, mais elle correspondit aussi à une pratique régulière, banalisée, de l'administration, s'étendant à l'ensemble de la société soviétique et non pas seulement à cette population. Dans le courant de l'année 1943, le Sovnarkom avait fait procéder à l'édition de 15 millions de passeports, témoignant bien qu'une très vaste opération de contrôle était en passe de s'effectuer. Les controverses de l'année 1942 sur l'attribution de la citoyenneté polonaise avaient aiguisé l'attention des autorités sur la diversité des statuts - apatride, polonais, soviétique — des amnistiés qui, au regard de l'ordre soviétique, nécessitait une intervention. D'ailleurs, en déterminant à trois ans la validité des passeports délivrés en 1940 dans les nouveaux territoires d'Ukraine et de Biélorussie, l'administration avait en quelque sorte programmé une nouvelle vérification des documents en 1943. Elle suivait de ce point de vue sa logique propre tout en répondant parfaitement à la demande politique.

Malgré l'état d'impuissance de l'ambassade, les derniers échanges au sommet entre Stalin, Molotov et Romer à propos des droits des résidents temporaires et permanents ne furent pas sans incidence sur le déroulement de l'opération. Celle-ci avait conduit, dans un premier temps, à reconnaître 4324 citoyens polonais. Mais une nouvelle directive du NKVD édictée le 3 avril ordonna d'attribuer également cette citoyenneté à tous les individus originaires de Pologne centrale. Suite à la révision de contrôle, près de 20000 personnes, à l'évidence anciens réfugiés arrivés dans les territoires annexés à l'automne 1939, obtinrent le statut ${ }^{82}$. L'arbitraire avec lequel les autorités soviétiques agissaient face à l'interlocuteur polonais n'empêchait pas néanmoins l'application d'une certaine légalité socialiste fondée sur le suivi des directives secrètes qui, depuis l'automne 1941, établissaient ses critères en conformité avec la position politique du moment.

Malgré la polarisation qu'elle entraîna sur la question de la citoyenneté, la banalité de la passeportisation s'exprimait, du côté de l'administration soviétique, dans le cynisme même avec lequel les agents procédaient. Ainsi ce responsable local qui, face à la résistance opposée par l'un des ex-déportés, tenta de le convaincre en ces termes : «Il n’y a pas de quoi faire une affaire de ce bout de papier ( $S_{\text {wistka }}$ papieru) qu'on te donne aujourd'hui, qu'on te reprendra demain et qui ne te coûte qu'un rouble. ${ }^{83}$

82. GARF, f. 9401, op. 2, d. 64, 1. 384-3. Cette révision donna lieu à une mention spéciale sur les passeports soviétiques alors qu'on laissa le passeport polonais aux 4000 personnes immédiatement reconnues comme citoyens polonais.

83. « Paszportizacja », art. cit.,p. 125. 
Au terme de la campagne près de 260000 personnes (257 660) avaient été identifiées et contrôlées. Cette estimation n'était, à vrai dire, pas très éloignée des dernières données élaborées par l'ambassade qui avait, de son côté, répertorié 263000 Polonais en 194384. Par rapport au nombre établi des 389382 anciens citoyens polonais présents en URSS lors de l'amnistie, et prenant en compte les départs de l'année 1942, l'administration centrale du NKVD reconnaissait que près de 12000 anciens citoyens polonais « étaient décédés ou avaient disparu pour d'autres raisons ${ }^{85}$. Parmi l'ensemble des personnes passeportisées, seuls les individus de nationalité ethnique juive (81 217 adultes et enfants) furent mentionnés dans la comptabilité, le nombre de personnes de nationalité biélorusse et ukrainienne n'étant pas cité.

Les résultats de la campagne de passeportisation confirmaient l'existence de quelques grandes concentrations d'anciens citoyens polonais, en particulier dans la république du Kazakhstan (77 000 personnes), de l'Ouzbékistan (25 500), dans les krai de l'Altaï (15 900), de Krasnoiarsk (14 200), les régions de Sverdlovsk (12 600) et de Tcheliabinsk (10 200), les autres étant dispersés dans 43 régions de l'URSS. La majorité était employée dans l'industrie (36\%) et dans l'agriculture (23\%), près de $11 \%$ l'étaient dans l'artisanat ; 7830 personnes (4\%) avaient été embauchées dans l'administration et près de $25 \%$ de l'ensemble se trouvaient sans emploi.

A priori, l'opération de passeportisation aboutissait, après de nombreuses tribulations, à l'intégration définitive des anciens déportés dans la société soviétique. Cette évidence doit néanmoins être largement relativisée à la lumière d'autres décisions prises dans la même période et dont le rôle allait s'avérer décisif dans le devenir des Polonais demeurés en URSS. En effet, la mise en place d'un nouvel encadrement à travers la création de l'Union des patriotes polonais reformula les termes d'une relation bilatérale, même si cette Union, animée par les communistes polonais, avait été fondée sur le territoire soviétique.

\section{L'ère des patriotes}

L'Union des patriotes polonais à laquelle se rattache étroitement la genèse du Comité polonais de libération nationale (Polski Komitet Wyzwolenia Narodowego - PKWN), fut officiellement créée en février 1943, suite aux pourparlers menés par Wanda Wasilewska avec les dirigeants soviétiques ${ }^{86}$. Sa création

84. K. Kersten, Repatriacja..., op. cit., p. 43.

85. GARF, f. 9401, op. 2, d. 64,1. 384-3.

86. Sur l'histoire de cette organisation, voir Z. Kumos, Związek Patriotów polskich, Varsovie, 1983; A. Głowacki, «Powstanie, organizacja i działalność Wydziału Opieki Społecznej Zarzadu Głównego Patriotów polskich w ZSRR (1943-1946) », Acta Universitatis Lodziensis, Łódź, 1981, p. 109-127 ; P. Żaron, Ludność polska w Związku Radzieckim w czasie II wojny światowej, Varsovie, PWN, 1990. A. Głowacki, Ocalić i repatriować. Opieka nad ludnościa polską w gtębi terytorium ZSRR, Łódź, WUI, 1994. Ces travaux éclairent de façon très inégale 
permit aux communistes polonais qui s'étaient regroupés à Lvov en 1939-1941, puis partiellement à Saratov, d'étendre considérablement le champ de leurs activités et de préparer la mise en place du futur État polonais. Le premier objectif de cette Union, formulé au cours de l'hiver 1943, était la formation d'une armée polonaise (future armée Berling), appelée aux côtés de l'Armée rouge à participer à la libération des territoires sous occupation allemande. La tâche de recrutement et d'organisation des contingents polonais ne constitua cependant qu'une partie des activités de l'Union car celle-ci se consacra plus généralement à la prise en charge des différentes communautés d'ex-déportés disséminées au sein de l'URSS. Le premier congrès de l'Union des patriotes polonais réunit plus d'une soixantaine de délégués en juin 1943, parmi lesquels figuraient, aux côtés de communistes convaincus comme A. Lampe, un certain nombre de personnalités politiquement « étrangères », tel Andrzej Witos, le frère du leader du parti paysan et ancien homme de confiance de l'ambassade, le socialiste Bolesław Drobner et bien d'autres dont la présence accréditait la volonté de rassemblement et d'unité des Polonais d'URSS sous la bannière de l'Union. Idéologiquement, cette organisation se présenta comme un vaste front antifasciste. Concrètement, elle se substitua en tant qu'autorité institutionnelle à la représentation « londonienne » qui venait de quitter l'URSS. L'Union des patriotes polonais reprit dès lors l'essentiel des activités déployées par l'ambassade à l'égard des populations civiles, à commencer par la distribution de l'aide humanitaire qui, d'abord conçue pour les familles de militaires, fut rapidement étendue à l'ensemble des communautés d'anciens citoyens polonais. Elle prit de même sous sa tutelle les institutions polonaises créées en URSS fin 1941 et confisquées en janvier 1943, qui comptaient, entre autres, plus d'une cinquantaine de jardins d'enfants, une trentaine d'écoles, 24 maisons d'invalides, 9 hôpitaux. En prenant progressivement en charge la gestion des Polonais, l'Union prit les attributs d'une véritable administration, appelée à recenser l'ensemble des « anciens citoyens ». Elle comptait une vingtaine de départements spécialisés, l'un des plus actifs étant le département d'aide sociale qui tissa à nouveau un réseau important d'hommes de confiance version « patriote », mais qui associa aussi d'anciens représentants de la période de collaboration polonosoviétique, Andrzej Witos ayant montré l'exemple, au sommet, d'une reconversion réussie ${ }^{87}$. Tout en s'étant inégalement implantée dans les différentes communautés d'anciens citoyens polonais, l'Union des patriotes put rapidement s'instituer comme leur organe de représentation. Fin 1943, elle avait créé des cellules locales dans 72 régions de l'URSS et avait procédé à l'enregistrement de 223800 personnes dont une majorité symbolique (51\%) était ethniquement polonaise, les autres comptant essentiellement des juifs (44\%), et une minorité de Biélorusses et Ukrainiens $(5 \%)^{88}$.

l'histoire de cette organisation. L'aide sociale, l'activité et le profil politique de ses dirigeants ont fait l'objet d'une attention soutenue, mais d'autres aspects, comme le personnel local de 1'Union, restent encore largement à étudier.

87. A. Głowacki, « Powstanie.... », art. cit.

88. P. Żaron, Ludność polska..., op. cit., p. 285. 
C'est en 1944 que l'Union atteignit son plein développement et connut un impact décisif même auprès des plus récalcitrants des anciens déportés. Son évidente inféodation à la direction stalinienne était aussi le gage d'une certaine marge d'action et son premier succès, de ce point de vue, fut d'obtenir des autorités soviétiques le transfert partiel des Polonais localisés dans les régions les plus austères du Nord de l'URSS, telle la république des Komis, vers les régions plus clémentes du Sud. Face au gouvernement central, la planification et le suivi de cette opération par l'Union constituèrent, de fait, un test de ses capacités d'organisation et de contrôle sur les populations concernées. Aux yeux des anciens citoyens polonais, cette initiative permit à l'Union de s'imposer comme une administration pro domo. Le principe de ces transferts, conçus à l'origine pour 26900 personnes, fut accepté par le Comité d'État à la défense, sur la proposition de l'Union des patriotes, ainsi que l'indiquèrent les décret du Sovnarkom relatifs à cette question, à commencer par celui du 18 mars 1944 qui avalisa la première décision sur le déplacement «en raison des conditions climatiques difficiles» des anciens citoyens polonais des régions du Nord vers les régions du Sud ${ }^{89}$. Ces premiers transferts visaient effectivement les collectivités localisées dans les républiques des Komis, de Iakoutie, d'Arkangelsk, et de Sibérie occidentale qui devaient être réparties en différents groupes comprenant au maximum 2000 personnes dans des kolkhozes des régions de Russie centrale et du Sud. La tâche de l'Union revenait à planifier des contingents, à coordonner les opérations entre régions de départ et régions de placement, toutes ces actions étant soumises au contrôle du pouvoir central qui, de son côté, fit procéder notamment aux vérifications des partants, ce qui conduisit à réduire le nombre de bénéficiaires. Ainsi en mai, lorsque Berija présenta à Stalin un premier état des lieux sur la question, seules 23250 personnes avaient été retenues sur les 26900 prévues $^{90}$. En revanche, les autorités locales se plaignirent souvent, aussi bien sur les lieux de départ que sur les lieux d'arrivée, de contingents en nombre plus important que ceux officiellement programmés ${ }^{11}$.

Malgré ces évidents dysfonctionnements, l'Union, une fois accepté le principe des transferts, ne cessa de solliciter les autorités centrales pour en élargir les quotas. Elle enregistra plusieurs échecs dans ses démarches, notamment dans ses tentatives de faire évacuer les Polonais de l'oblast' de Djambul au Kazakhstan, les dirigeants républicains s'y étant fortement opposés, arguant de la pénurie de main-d'œuvre qui s'ensuivrait. En guise de compensation, le Sovnarkom de l'URSS ordonna l'envoi de 500 tonnes de farine pour les anciens citoyens polonais ${ }^{92}$. En juillet, l'Union adressa une nouvelle requête concernant 45000 personnes jusque-là majo-

89. GARF, f. 5446, op. 46a, d. 261,1.2-3.

90. GARF, f. 9401, op. 2, d. 64, 1. 384ob. Sur les 23250 individus sélectionnés, seul le placement de 9000 était alors concrètement envisagé dans les régions de Voronej, Rostov, Stavropol, etc. Les autres furent par la suite évacués vers l'Ukraine centrale et l'Ukraine du Sud.

91. GARF, f. 5446, op. 46a, d. 261, 1. 21-22 ; d. 232, 1.7 ; d. 205, 1. 2 ; Archivum Akt Novyh (cité infra AAA), Związek patriotów polskich (infra ZPP), sygn. 216/24, k. 38.

92. GARF, f. 5446, op. 46a, d. 205,1.1-5. 
ritairement employées dans l'industrie forestière, en proposant que cette population soit placée dans les sovkhozes d'Ukraine centrale où « elle pourrait efficacement contribuer au redressement de l'agriculture dans la république $»^{93}$. Le Sovnarkom de l'URSS retint le chiffre de 30000 personnes, mais par la suite il s'avéra que près de 33000 furent effectivement replacées en Ukraine et cette opération, qui entraîna de nombreuses difficultés logistiques, concentra l'essentiel de l'énergie déployée dans le suivi des transferts qui se déroulèrent à partir de l'été $1944^{94}$.

D'après les nombreux rapports établis sur ces déplacements internes, ceux-ci étaient apparentés, dans l'esprit des dirigeants, aux très vastes mouvements de réévacuation des Soviétiques, réfugiés dans les territoires de l'Est à partir de juin 1941. Les documents relatifs aux transferts des Polonais les qualifient d'ailleurs d' «évacués ». Mais contrairement aux Soviétiques de Leningrad, de Moscou et d'autres régions, il n'est pas question de les relocaliser dans leurs terres natales. La plupart a été placée, par petits groupes, dans les sovkhozes de Kherson, Nikolaevsk, Dniepropetrovsk, Kharkov, Odessa, etc., chacune de ces régions ayant accueilli quelques milliers d'entre eux (2 000 en moyenne mais jusqu'à 4500 dans les oblasti du sud de la république comme à Kherson) ${ }^{95}$. Autrement dit, ces opérations correspondaient à un placement de main-d'œuvre dans des zones déficitaires, libérées seulement quelques mois auparavant, qui pouvaient servir, de tous ces points de vue, de test d'intégration à long terme des Polonais, ce que suggérait également l'argumentaire présenté par l'Union sur l'intérêt des transferts. Mais le contexte plus large du milieu de l'année 1944 conduit surtout à envisager cette migration comme une étape dans le processus de « rapatriement » des ex-déportés vers la Pologne.

\section{Des transferts frontaliers aux évacuations des « nouveaux » citoyens polonais}

Parallèlement à la réalisation, entre mai et l'automne 1944, des transferts internes, la nouvelle donne sur le front de l'Ouest accéléra le processus d'externalisation des Polonais soviétisés.

La contribution de l'armée polonaise - qui comptait 36510 membres en mai 1944 - aux offensives de l'Armée rouge à l'ouest eut en particulier pour effet de réactualiser la question de la citoyenneté de ces militaires, parvenus en Pologne orientale en libérateurs. C'est à leur intention que fut édicté l'ukaz du Présidium du Soviet suprême du 22 juin 1944 relatif aux droits d'acquérir la citoyenneté polonaise. Ce document mentionnait les militaires de nationalité polonaise et leurs familles, résidents d'Ukraine et de Biélorussie occidentale au 29 novembre 1939, ainsi que les individus ayant contribué à la lutte pour la libération de la Pologne ${ }^{96}$.

93. Ibid., d. 261,1. 18-25.

94. Ibid., 1.25 et sq.; f. 5446, op. 47a, d. 254, 1-6.

95. Ibid.

96. GARF, f. 7523, op. 10, d. 69a, 1.3-4. 
La procédure indiquée se référait implicitement à la loi du 12 août 1938 en précisant que le changement de citoyenneté s'effectuait par requêtes à adresser directement ou via le commandement militaire polonais à la commission ad hoc ${ }^{97} \mathrm{du}$ Présidium du Soviet suprême de l'URSS, sachant - précisait l'ukaz - que celle-ci comprenait des membres de l'Union des patriotes polonais (UPP) ${ }^{98}$. Ceux-ci furent donc directement associés à la prise de décision sur les attributions de la citoyenneté polonaise ${ }^{99}$.

L'exclusion, parmi les ayants droit, des « Polonais-Lituaniens », prononcée en mai 1942 par le vice-commissaire du NKID, fut abolie par un ukaz du Présidium du 14 juillet 1944 qui étendait le bénéfice des conditions d'accès à la citoyenneté polonaise aux résidents de Lituanie ${ }^{100}$. Cette concession, première d'une série, annonçait déjà l'abolition des clauses conditionnelles introduites au cours des années 1941-1942.

La possibilité, réactualisée, d'acquérir la citoyenneté polonaise fut néanmoins abordée de façon très sélective. Conçue dans une optique méritoire, elle revêtait, du moins à l'égard des civils combattant pour la liberté, un caractère discrétionnaire et témoignait, en tout cas, de la prudence avec laquelle les Soviétiques envisageaient alors l'éventualité pour les anciens déportés de partir vers la Pologne. L'attitude à l'égard des Polonais soviétisés, qui confirmait en quelque sorte l'absence de perspectives clairement formulées sur leur devenir, contrasta de façon singulière avec la décision prise, à peine deux mois plus tard, de procéder aux échanges « ethniques » de populations frontalières. Les accords passés en septembre 1944 entre les républiques soviétiques d'Ukraine, de Biélorussie et de Lituanie, d'une part, et le Comité polonais de libération nationale (PKWN), de l'autre, prévoyaient en effet le transfert vers la Pologne des populations de nationalité polonaise et juive résidentes des régions frontalières ainsi que celui des Ukrainiens, Biélorusses, Russes et Ruthènes résidents des régions frontalières de Pologne vers les républiques soviétiques ${ }^{101}$.En se basant sur la localisation, en septembre 1944, des populations concernées, les accords excluaient de fait les anciens déportés. L'objectif poursuivi d'homogénéisation ethnique des territoires contestés était clairement d'anticiper le changement

97. La Commission «pour l'examen des questions d'entrée, de réception et d'exclusion de la citoyenneté soviétique » avait fait l'objet d'une réorganisation par décision du 23 novembre 1943. Elle était désormais dirigée par A. F. Gorkin (secrétaire du Sovnarkom), et comprenait S. Kruglov (NKVD, vice-commissaire), N. Pugovkin (Narkomjust, vice-commissaire), A. P. Pavlov (NKID, membre du collège), Beljaev (NKID, chef du département consulaire), GARF, f. 7523, op. 10, d. 67,1.35.

98. GARF, f. 7523, op. 10, d. 69a, 1.3-4.

99. Entrèrent dans la commission H. Wolpe, A. Juszkiewicz et I. Kuczynska. AAA, ZPP, présentation historique.

100. GARF, f. 7523, op. 10, d. 69a, 1. 5. Mais contrairement aux notes de mars et mai 1942 qui concernaient tous les individus de nationalité polonaise, anciens citoyens des républiques baltes, l'ukaz de 1944 ne se référait qu'aux Polonais de Lituanie qui étaient, de fait, les seuls sauf exception à être effectivement concernés par la question.

101. GARF, f. 9415, op. 3, d. 1400,1. 8-44, accord du 9 septembre entre le PKWN et la république d'Ukraine, accord du 9 septembre entre le PKWN et la république de Biélorussie, accord du 22 septembre entre le PKWN et la république de Lituanie. 
officiel du tracé des frontières en plaçant les acteurs nationaux et internationaux devant le fait accompli. Mais cette décision prise avec le PKWN, ce comité prosoviétique issu en partie de l'Union des patriotes polonais et, à cette date, pratiquement inconnu en Pologne même, qui intervenait alors qu'une partie importante du territoire polonais était encore occupée par les Allemands, fut loin d'être immédiatement effective ${ }^{102}$. Il faudra dans les faits plus de deux ans pour réaliser le transfert de plus d'un million de personnes vers la Pologne ${ }^{103}$.

Les accords de septembre 1944 témoignaient, après l'ère des restrictions ethniques, d'un changement important dans la conception des populations polonaises auxquelles étaient désormais associés les juifs. Cet élargissement, qui mériterait d'être resitué dans le contexte plus vaste de l'appréhension de la question des juifs par le pouvoir soviétique, fut lié, dans le cas des Polonais, à l'influence de l'Union des patriotes. L'organisation, qui comptait de nombreux juifs à la fois parmi ses représentants et ses simples membres, venait, en juillet 1944, d'officialiser leur présence par la création au sein de l'appareil central d'un « Comité d'organisation des juifs polonais en URSS ». Ce comité joua un rôle important surtout auprès des fondations juives étrangères dont il sollicita le soutien ${ }^{104}$. Mais il avalisait aussi en la portant au grand jour l'existence de juifs «polonais »dont l'attribut avait été jusque-là dénié, et qui allaient désormais systématiquement figurer dans les ordres d'évacuation vers la Pologne ${ }^{105}$.

Les nombreuses instructions qui suivirent les accords de septembre 1944 pour l'enregistrement des candidats au départ et l'accueil des nouveaux arrivants ne contenaient aucune indication concernant le changement de citoyenneté, fait qui ne sera relevé que plus d'un an après, en octobre 1945, par le vice-commissaire du NKVD, qui était alors inquiet surtout des modalités d'enregistrement des Ukrainiens transférés en URSS de Pologne ${ }^{106}$.

102. Le 11 décembre 1944, seules 4200 personnes avaient été effectivement évacuées d'Ukraine et les rapports périodiques présentés au CC du PC ukrainien dans cette période insistaient tous sur la réception très défavorable de la perspective des transferts, conçus théoriquement sur la base du volontariat. Central'nyj gosudarstvennyj arhiv obščestvennyh ob"edinenij Ukrainy (infra CGAOOU, Kiev), f. 1, op. 23, d. 790,1. 216 (pour le bilan).

103. Sur 1'histoire de ces transferts, voir notamment, K. Kersten, Repatriacja... op. cit.; J. Czerniakiewicz, Repatriacja ludności polskiej z ZSRR w latach 1944-1948, Varsovie, 1987; S. Ciesielski, Przesiedlenie ludności polskiej z kresów wschodnich do Polski 1944-1947, Varsovie, Neriton Pan, 1999.

104. A. Głowacki, Ocalić..., op. cit., p. 55. Le fonds de l'UPP ne contient aucun dossier sur ce comité et la présentation de l'inventaire ne fait que mentionner son existence sans apporter aucun élément substantiel d'information.

105. Selon les bilans généraux des évacuations frontalières, 4837 juifs partirent de Biélorussie parmi les 231152 Polonais évacués ; 2284 juifs partirent de Lituanie sur un total de 171158 évacués ; 810415 personnes furent évacuées d'Ukraine, mais les autorités républicaines ne produisirent aucune statistique concernant le nombre de juifs que ce chiffre comprenait, GARF, f. 9415 , op. 3c, d. 1415, 1.92-93.

106. GARF, f. 9415, op. 3, d. 1400,1.6. Les formulaires d'enregistrement ne comprenaient pas de rubrique sur la citoyenneté 
Cette «négligence » soulignait, en quelque sorte, la distinction faite par les autorités soviétiques entre les populations polonaises tout juste libérées de l'occupant allemand et les « anciens citoyens polonais » passeportisés dont la procédure de sortie allait se révéler, un an plus tard, d'une extrême complexité. À l'automne 1944, il n'est cependant pas encore question de leur « rapatriement » vers la Pologne. Seules, les autorités d'Ukraine qui venaient de procéder au placement de 33000 personnes venues de l'Est plaidaient déjà pour leur départ, arguant de leur faible productivité et de leur comportement 《antisoviétique »107. Dans l'hypothèse où ces premiers transferts auraient été conçus comme un test pour le replacement durable des anciens déportés, l'échec s'avérait patent, du moins aux yeux des autorités républicaines qui ne cachèrent pas, dans ce cas de figure comme dans celui plus général des transferts frontaliers, leur volonté d'homogénéiser l'Ukraine par l'expulsion des minorités polonaise et juive ${ }^{108}$.

À l'automne 1944, dans l'euphorie de l'annonce de la libération de Varsovie par l'Armée rouge, l'Union des patriotes polonais déploya une intense propagande auprès des communautés restées en URSS. Essentiellement destinée à populariser l'action du PKWN et à le présenter comme l'instance gouvernementale de la nouvelle Pologne, cette propagande apportait un contenu patriotique à la version soviétique des tragédies de la guerre et de la libération. C'est à cette époque que furent organisées, comme à Karaganda, d'importantes collectes d'argent pour l'édification de monuments en mémoire des officiers polonais « assassinés à Katyn par les nazis »109. La multiplication de bulletins locaux, d'émissions de radio, de conférences périodiques sur la «consolidation de la Pologne démocratique » accompagna cette période de sortie de la guerre au cours de laquelle les anciens déportés furent désormais assimilés par les représentants de l'UPP à des « émigrés ». Le développement des activités politiques et culturelles des communautés, l'encadrement des enfants par la création d'organisations apparentées aux komsomols, témoignaient, au-delà de la citoyenneté formelle, d'une soviétisation des esprits, stimulée par la liesse de la libération et l'atmosphère unitaire qui l'accompagna. Quant à la perspective du retour, elle ne se fit jour, parmi les anciens déportés, que dans les premiers mois de l'année 1945.

À partir de décembre 1944, l'Union des patriotes polonais fut appelée à créer une nouvelle cellule à Lvov pour participer à l'organisation des transferts des résidents frontaliers, tâche qui annonçait déjà son rôle à venir dans l'organisation des

107. GARF, f. 5446, op. 47a, d. 254, 1. 33-35 ; CGAOOU, f. 1, op. 23, d. 1466, 1. 1-6. Ce dernier rapport de décembre 1944 précisait par « comportement antisoviétique » que ces Polonais reconnaissaient tous l'autorité du gouvernement de Londres.

108. Le rôle des dirigeants ukrainiens dans la décision d'évacuer l'ensemble des populations déportées est une hypothèse qui entraînerait de trop longs développements mais qui mérite néanmoins de figurer, sachant que la politique conduite à l'égard des minorités polonaise et juive a été très différente selon les autorités républicaines concernées, celles d'Ukraine ayant eu l'attitude la plus radicale. S. Ciesielski, Przesiedlenie..., op. cit.

109. AAA, ZPP, sygn. 112, k. 33-35. 
rapatriements des « émigrés ${ }^{110}$. Fin janvier 1945 , la conférence tenue par l'Union à Moscou aborda longuement la question du nouveau tracé des frontières orientales de la Pologne, fournissant aux représentants locaux l'argumentaire nécessaire à la justification de la perte de ces territoires et à l'idée d'un retour, non dans la terre natale, mais « dans les terres de la Pologne ancestrale $»^{111}$.

\section{L'actualité des retours}

Contrairement aux accords signés en septembre 1944 à l'échelle des autorités républicaines et du PKWN pour le transfert des populations frontalières, le nouvel accord du 6 juillet 1945, passé entre l'URSS et le Gouvernement provisoire d'unité nationale de la république de Pologne, s'inscrivait dans le cadre d'une relation étatique bilatérale clairement établie dans les textes. Il rehaussait en les confirmant l'autorité des décisions prises près d'un an auparavant sur les « évacuations » et introduisait dans la définition des bénéficiaires un nouveau paragraphe concernant « les individus de nationalité polonaise et juive, citoyens polonais jusqu'au 17 septembre 1939 et résidents des autres régions de l'URSS » (les premiers bénéficaires étant, rappelons-le, les résidents des régions occidentales des républiques frontalières) ${ }^{112}$. L'accord du 6 juillet conduisit à la création rapide d'une Commission bilatérale chargée des évacuations ${ }^{113}$.

La voie était désormais ouverte pour l'organisation des retours, du moins théoriquement, car le processus de sortie de l'URSS représenta pour les anciens déportés une nouvelle course d'obstacles imprévisibles. Alors que les populations frontalières furent surtout confrontées à la suspicion obsessionnelle de « nationalisme » lors des contrôles au départ, les ex-déportés eurent principalement à affronter, une fois encore, la tyrannie administrative de l'enregistrement et de l'exigence de preuves identitaires. L'accord sur les évacuations fut, en effet, immédiatement précédé le 5 juillet de l'ukaz du Présidium du Soviet suprême sur le droit des individus de nationalité polonaise et juive « à sortir de la citoyenneté soviétique » qui, dans son paragraphe 3 , réitérait l'obligation de présenter la requête à la commission

110. Dans cette période, la grande difficulté des autorités ukrainiennes était la présence, en nombre infime, de membres des délégations polonaises locales chargées d'enregistrer les candidats au départ et qui s'avéraient politiquement peu fiables. CGAOOU, f. 1, op. 23, d. 1465 , 1. 18-20 ; d. 1466,1. 7-16. AAA, ZPP, sygn. 141, k. 41-46.

111. AAA, ZPP, sygn. 141, k. 14-15.

112. GARF, f. 9415 , op. 3, d. 1400,1.59-64.

113. GARF, f. 5446, op. 47, d. 63, 1. 4-7 ; f. 5446, op. 47a, d. 374, 1. 10-12. Le fonctionnement de cette commission bilatérale était en réalité très inégalitaire car elle accordait aux Soviétiques un droit de regard sur le fonctionnement de la délégation polonaise qu'elle ne reconnaissait pas à l'autre partie. Figuraient, dans la délégation soviétique de la commission bilatérale, Kosygin (vice-président du Sovnarkom), Alexandrov (président de la délégation soviétique de la commission), Galkin (responsable de l'administration centrale de la milice du NKVD), Dobrovolskij (représentant du Narkomfin), Sarov (représentant du Narkomtorg), Miterev (chargé des questions sanitaires), Arutjunov (chargé de la logistique des transports), Ljubimov (chargé de l'approvisionnement des évacués). 
du Soviet surpême de l'URSS ${ }^{114}$. D'après le texte, la procédure s'appliquait à l'ensemble des candidats à l'évacuation, mais dans les faits elle ne concerna que les anciens déportés, les formalités administratives de départ des populations frontalières ayant été déjà définies et mises en pratique depuis l'automne 1944115. L'application de l'ukaz du 5 juillet 1945 revenait à procéder à une vaste opération de dépasseportisation.

De juillet à décembre 1945, les modalités de «sortie de la citoyenneté soviétique » donnèrent lieu à de très nombreuses correspondances transformant, au fur et à mesure des consultations, instructions et contre-instructions, la procédure élaborée. Dans un premier temps, c'est le NKID, en collaboration avec la commission du Présidium du Soviet suprême, qui, début août, définit la marche à suivre. Les compétences dans l'organisation et le suivi de la procédure revenaient au NKVD. Les instructions relatives à la formulation des demandes mettaient l'accent sur la nécessité de fournir à l'appui de la requête des documents prouvant l'ancienne sujétion polonaise et qui pouvaient être de nature très diverse, allant du passeport soviétique - s'il était mentionné qu'il avait été fourni sur la base d'un passeport polonais - jusqu'à des attestations de fin d'études, récépissés de paiement d'impôts, livrets sanitaires et autres ${ }^{116}$. Aucun paragraphe n'abordait la question de la nationalité (polonaise ou juive) des ayants droit, ceux-ci n'étant définis que par référence à leur ancienne citoyenneté. Ainsi, toutes les distinctions ethniques, si préjudiciables trois ans auparavant, devenaient lettre morte dans le nouveau contexte étatique bilatéral, confirmant a posteriori leur caractère éminemment circonstanciel et instrumental.

Le 24 août un prikaz de Berija établit l'ordre interne de la procédure, transmise sous forme d'instructions détaillées à toutes les administrations locales en charge du dépôt des demandes. Celles-ci devaient transmettre à l'administration centrale de l'OVIR (Otdel viz i registracija inostrannyh graždan - Département des visas et d'enregistrement des citoyens étrangers) des listes nominatives mentionnant précisément les références des documents fournis dans les milices locales à l'appui des requêtes. Cependant, les instructions insistaient surtout sur l'efficacité attendue des responsables locaux, l'examen des demandes devant être achevé avant le $1^{\text {er }}$ novembre $1945^{117}$. Cette exigence d'efficacité fut sans doute à l'origine de la légèreté manifestée dans l'application des consignes relatives à la preuve identi-

\section{GARF, f. 7523, op. 10, d. 69a, 1. 8-9.}

115. CGAOOU, f. 1, op. 23/1, d. 790, 792, 793, 1464, 1466, 1467. Rappelons qu'en Ukraine d'où partirent la majorité des évacués frontaliers, les transferts concernèrent d'abord la population rurale, enregistrée sur des listes établies par des délégués ad hoc, et ce principe d'enregistrement dès lors prévaudra. Les nombreux documents relatifs à l'application de la procédure ne mentionnent jamais, sauf cas exceptionnel, de problèmes relatifs à l'identification des personnes. En revanche, l'enregistrement des biens des évacués fait l'objet de nombreuses correspondances. Ces remarques ne valent cependant que pour l'Ukraine, les autorités des autres républiques, en particulier de Lituanie, ayant mené des politiques très différenciées dans la question des transferts.

116. GARF, f. 5446, op. 47a, d. 374, 1. 5-10.

117. GARF, f. 9415 , op. 3, d. 1400, 1. 65-66. 
taire, du moins à en croire les récriminations que fit l'administration centrale à ce sujet début octobre. En effet, nombre de listes envoyées à l'OVIR ne portaient aucune indication des documents présentés et devaient pour cette raison être réexpédiées pour compléments ${ }^{118}$. En rappelant la nature des documents exigés, le responsable précisait qu'ils devaient être traduits en russe et indiquait que les représentants de l'Union des patriotes polonais étaient mandatés pour ce faire. Jusqu'alors l'UPP n'avait été explicitement sollicitée, dans les formulaires des requêtes, que pour attester du bien-fondé des demandes des « combattants pour la liberté » présentées à la commission centrale ${ }^{119}$. Elle l'était désormais pour les demandes auprès de l'administration locale.

Après avoir mis en cause le travail effectué au niveau de l'enregistrement, il s'avéra qu'en fait la production de la preuve constituait un problème insoluble dans bien des cas et la centralisation de la procédure ne conduisait dès lors qu'à un va-etvient de courriers sans lendemains. Pour remédier à l'impasse bureaucratique ${ }^{120}$, le Sovnarkom en vint à modifier radicalement la procédure en exigeant des autorités républicaines la constitution de commissions spéciales pour l'examen des demandes ${ }^{121}$. Un mouvement de décentralisation s'amorçait pour rendre effectif le contrôle administratif. D'après les directives du NKVD qui accompagnèrent cette réorientation, la décentralisation présentait, entre autres, l'intérêt de permettre de statuer sur les cas des «sans-papiers » en recourant aux archives des milices locales ${ }^{122}$. L'administration centrale en venait par là à reconnaître sa propre responsabilité dans la quadrature du cercle à laquelle elle se trouvait confrontée. Comment, en effet, exiger des preuves documentaires d'individus dont les documents avaient été précédemment confisqués ? Les directives du NKVD relatives à la création des commissions spéciales concernaient longuement les «sanspapiers » et indiquaient précisément les démarches à suivre : utiliser les matériaux des organes du NKVD sur la passeportisation de 1943 ou recourir aux rapports élaborés sur les lieux administratifs de détention lors de l'amnistie d'août 1941.En cas d'absence de toute trace dans les administrations locales, des recherches devaient alors être engagées dans le Premier département spécial (specotdel) du NKVD de l'URSS. S'agissant des personnes «documentées» (dokumentirovannye ljudi), il était bien précisé, cette fois-ci, de ne pas confisquer leurs papiers

118. Ibid., 1. 69. Sur 7180 demandes adressées à l'OVIR, 1141 avaient dû être renvoyées pour compléments. Les administrations des milices d'Ouzbekistan, du Daghestan, d'Ukraine, d'Azerbaidjan, étaient explicitement mises en cause.

119. C'est-à-dire les civils mentionnés dans la loi de juin 1944.

120. La consultation des archives de l'OVIR fournirait un éclairage complémentaire important sur la nature de cette impasse, sans doute également redevable au fonctionnement de cette administration.

121. GARF, f. 7523 , op. 16, d. 57,1.1-2 ; f. 9401 , op. 2, d. 168, 1. 391. Ces commissions devaient comprendre le vice-président de l'Ispolkom (dans le cas où les commissions pouvaient être créées au niveau régional) ou du SNK de la République fédérée, l'adjoint au chef de l'administration du NKVD, l'adjoint au chef de l'administration du NKGB, l'adjoint au procureur, de la région, du kraj ou de la république selon les cas.

122. GARF, f. 9415 , op. 3, d. 1400, 1. 70-74. 
mais d'en faire des copies ${ }^{123}$. Enfin les commissions étaient sommées de rendre compte de leur activité et de fournir les listes de sans-papiers.

\section{La dépasseportisation effective}

La vigilance attendue des commissions dans la production de la preuve était toujours officiellement rappelée début décembre 1945 dans les projets de directives du NKVD soumis à la décision de Kosygin. Mais en fait un changement notoire était en passe de s'effectuer à travers l'introduction d'un nouveau paragraphe indiquant de confier aux commissions spéciales la tâche de statuer sur les sans-papiers, selon leurs convictions (usmotrenie) ${ }^{124}$. La décision administrative était donc entièrement déléguée aux instances régionales et républicaines, elle mettait fin au contrôle exercé par le centre sur le fonctionnement des administrations locales et marquait le passage à la phase massive de vérification et de sélection des partants ${ }^{125}$. La latitude laissée aux commissions pour statuer sur les citoyens polonais n'impliquait pas, cependant, l'abandon de l'exigence de preuves, instrument de pression et de confrontation efficace, bien ancré dans les pratiques normatives de contrôle du NKVD et plusieurs fois expérimenté à l'égard des " anciens citoyens polonais ».

S'agissant de la prise de décision locale et des interlocuteurs en présence, le rôle de l'Union des patriotes polonais doit être rappelé car, bien que le recours à cette organisation à titre de caution pour les sans-papiers n'ait pas figuré dans les directives centrales, elle remplit effectivement cette tâche, comme l'indiquaient les instructions internes de son comité directeur qui précisaient que, dans ce cas de figure, le témoignage de deux anciens citoyens polonais, confirmé par l'agent local de l'UPP, pouvait pallier le défaut de preuve ${ }^{126}$. À l'évidence, l'adhésion à l'Union favorisait l'obtention de l'attestation, quand elle n'était pas conditionnée par cette démarche. C'est au cours de cette période que l'Union enregistra le plus de membres, soit environ $70 \%$ de la population adulte ${ }^{127}$. Faute d'être reconnue officiellement, l'autorité du document était, elle, fonction des relations entre les agents de l'UPP et l'administration locale qui, tout en étant inégales, se caractérisaient néanmoins dans la majeure partie des cas par une active collaboration ${ }^{128}$. La voie était désormais ouverte pour considérer peu ou prou que les membres inscrits dans les listes de l'Union étaient des anciens citoyens polonais et vice versa, ce qui

123. Ibid., 1.73

124. Ibid., 1.77 .

125. À la date du 29 octobre, seuls 12934 dossiers avaient été présentés pour examen, le 20 décembre on en comptait 56 052. GARF, f. 9415 , op. 3, d. 1400,1. 69.

126. AAA, ZPP, sygn. 8, k. 28.

127. A. Głowacki, Ocalić..., op. cit., p. 59.

128. Les archives des représentations locales de l'UPP sont très disparates et inexistantes dans certains cas, ne permettant pas de ce fait de se faire une idée exacte des différentes situations locales. C'est en Ukraine orientale que des entraves ont été mises à l'activité de l'Union. Voir à ce propos AAA, ZPP, sygn. 19. 
permettait aux administrations de se délester, en partie du moins, des tâches de contrôle désormais urgentes. Les pouvoirs concédés aux commissions s'inscrivaient en effet dans un contexte nouveau de pressions au sommet pour la réalisation des rapatriements. Le «changement d'opinion»sur les évacuations évoqué, le 14 décembre, lors de la réunion des membres soviétiques de la Commission bilatérale, n'en précisait ni l'origine ni la nature, mais les consignes passées ce jour-là revenaient à formuler l'ordre impératif d'accélérer la procédure d'évacuation ${ }^{129}$.

Sans préjuger des motifs spécifiques de ce «changement» concernant les « anciens citoyens polonais », on peut néanmoins le rapprocher d'autres mesures prises dans la même période à l'égard de certaines populations, comme les prisonniers de guerre du camp allié ou les civils internés, dont les délais de rapatriement avaient été également fixés à la fin de l'année 1945. Or on constate pour les prisonniers de guerre ce même revirement visant soudainement à achever les rapatriements ${ }^{130}$. En cette fin d'année, la direction stalinienne procédait ainsi à un premier bilan général de cette tâche, à vrai dire immense, d'organisation des migrations de retours et prenait acte de la relative paralysie de son administration. Le non-respect des délais était sur le point de constituer une faute grave, il s'agissait bien de mettre fin à la période flottante de sortie de guerre.

Dès lors, parallèlement à la vérification des individus, la planification des retours se concrétisa rapidement, tout en se heurtant encore à des obstacles bureaucratiques. Ainsi, Alexandrov, qui avait été chargé de pourvoir aux formulaires d'autorisation de sorties du territoire et qui avait fait éditer 240000 documents $^{131}$, se vit obligé par le NKVD de modifier les directives affairantes à leur diffusion, Černišov exigeant qu'y figurent des photos d'identité visées par tampon de l'administration locale ${ }^{132}$. Malgré toutes sortes de dysfonctionnements, l'ordre signifié d'en haut apporta vite des résultats probants. Au 31 décembre 1945, 100830 demandes avaient été présentées pour examen. Seul le Kazakhstan se distinguait par l'absence de toute donnée sur la conduite des vérifications ${ }^{133}$. Dix jours plus tard, le nombre s'élevait à 159550 , mais les commissions n'avaient statué que sur 66900 cas $^{134}$. Le 11 janvier 1946, les délégués soviétiques de la Commission centrale bilatérale prirent de nouvelles mesures pour accélérer, parallèlement aux contrôles des individus, la mise en œuvre des rapatriements, 60000 départs ayant été alors programmés pour le $1^{\mathrm{er}}$ mars ${ }^{135}$. À cette date,

129. GARF, f. 9415 , op. 3 , d. 1400,1.80.

130. Voir à ce propos, C. Klein-Gousseff, ed., Retours d'URSS. Les prisonniers de guerre français et les internés dans les archives soviétiques, 1941-1950, Paris, CNRS-Éditions, 2000.

131. D'après un rapport statistique du NKVD de février 1947, le nombre d'anciens citoyens polonais présents en URSS fut estimé en janvier 1946 à 220000 et, de fait, c'est ce chiffre qui sera effectivement retenu et non les 240000 avancés d'après le nombre de formulaires édités. GARF, f. 9401,op. 2, d. 168,1.399.

132. GARF, f. 9415 , op. 3 , d. $1400,1.84$

133. Ibid., 1.87

134. Ibid., 1. 88 .

135. Ibid., 1. 92 . 
170000 demandes sur les 180000 présentées avaient été contrôlées par les commissions et 34480 citoyens polonais étaient repartis vers leur patrie non sans avoir, au préalable, rendu leur passeport soviétique, du moins pour ceux qui le possédaient ${ }^{136}$. Tandis que l'efficacité des contrôles des commissions était désormais statistiquement attestée et que les rapatriements commençaient à devenir effectifs, la délégation soviétique reçut l'annonce de la libération des anciens citoyens polonais détenus dans les prisons et les camps pour lesquels elle fut appelée à définir, à nouveau, les procédures de sortie. Tout en reconnaissant que la majorité des détenus étaient des individus « sans citoyenneté », Kosygin, qui avait été consulté à ce sujet par la délégation, conclut qu'il fallait assimiler ce nouveau contingent aux autres citoyens polonais visés par l'accord du 6 juillet 1945 et ne pas engager de procédure de vérification spécifique à leur égard ${ }^{137}$. La volonté d'achever le plus rapidement possible les rapatriements prévalait décidément sur la préoccupation de contrôle qui avait marqué la conception et la mise en œuvre initiales du processus. L'impératif d'efficacité se traduisit, dans les rapports périodiques sur l'état comptable de la dépasseportisation et des rapatriements, par des résultats spectaculaires puisqu'entre décembre et juin 223000 personnes avaient été « vérifiées » et 222000 étaient parties. En ajoutant à ces résultats la part des militaires, la somme des évacuations correspondait en fait approximativement au bilan de la passeportisation de 1943 qui avait ainsi fourni, dans un objectif a priori contraire, les paramètres de cette dernière opération. À ce stade, la délégation soviétique de la Commission bilatérale décida que les évacuations étaient achevées et la fermeture, en juillet 1946, des bureaux moscovites de l'Union des patriotes polonais sanctionna cet état de fait.

\section{Conclusion}

Au fil de cette rétrospective, la citoyenneté, telle qu'elle a été imposée et appliquée par les autorités soviétiques, se présente non comme un concept qui définirait le lien de l'individu à l'État, mais davantage comme une série de mesures administratives aux critères changeants, traduisant la position politique de la direction stalinienne à l'égard des régions conquises, et plus généralement, sa volonté de contrôler une nouvelle population.

Dans la période genèse de l'annexion, c'est une approche totalisante de la citoyenneté qui domine ; elle repose sur la territorialisation des individus dans les régions incorporées. Aucune distinction explicite n'a été introduite dans la qualité de résident (permanent ou temporaire) qui permettrait d'envisager une conception de la citoyenneté basée sur la loi du sol, défini par le lieu de naissance, voire par la résidence permanente. Dans son principe, l'ukaz du 29 novembre 1939 proclamait soviétiques toutes les personnes présentes, affirmant ainsi la mainmise totale de l'URSS sur ses nouveaux territoires.

136. Ibid.,1. 118.

137. Ibid.,1. 125. 
À partir de l'automne 1941, une exception est introduite permettant aux Polonais ethniques, originaires des régions conquises en 1939, d'être soustraits à la citoyenneté soviétique, celle-ci se fondant donc désormais sur les critères d'appartenance nationale des individus. Mais la logique à l'œuvre, qui pourrait s'apparenter à la loi du sang, ne s'est manifestée que partiellement. En admettant que les Biélorusses et les Ukrainiens, en tant que natifs des régions annexées aux républiques nationales d'Ukraine et de Biélorussie soviétiques, dussent être reconnus comme soviétiques, aucun fondement ethnico-national ne pouvait en revanche justifier l'appropriation par l'État soviétique de la population juive, position sur laquelle d'ailleurs il reviendra. L'utilisation de l'argument ethnique est donc surtout apparue pour rappeler l'illégitimité des revendications territoriales polonaises et plus pragmatiquement pour affirmer le droit de contrôle soviétique sur la sélection des ayants droit à changer de citoyenneté.

La campagne de passeportisation de 1943 a mis théoriquement fin à l'état d'exception de 1941 sans pour autant revenir à la position de l'ukaz de 1939 puisque désormais près de 20000 personnes étaient reconnues comme citoyens polonais en vertu de leur lieu de naissance (la Pologne centrale et non les territoires annexés). Le gouvernement soviétique en venait-il tardivement à faire reposer le critère de citoyenneté sur le droit du sol ? La réponse fournie par l'évolution ultérieure est évidemment négative, et le sens même de cette opération de passeportisation apparaît a priori assez contradictoire dans son contexte immédiat, puisque deux intentions opposées étaient affichées : celle d'intégrer une population par la confirmation de sa sujétion soviétique, celle de la maintenir en situation d'extériorité par la création d'un nouvel organe de médiation. Mais cette opposition ne vaut que si l'on prête à l'intention un projet clairement défini, ce qui n'était pas le cas. Perçues à travers leurs objectifs très pragmatiques, les décisions de 1943 paraissent, en fait, assez cohérentes au regard du tournant politico-militaire amorcé dans la période et compte tenu de l'émergence de nouveaux interlocuteurs polonais. La campagne de (re)soviétisation s'est présentée à la fois comme un message politique de rupture à l'égard de l' " allié » polonais et comme une opération de bilan de la situation des amnistiés après les départs de 1942. L'action administrative de 1943 a ainsi permis de dresser un état des lieux statistique de cette population (localisation, composition ethnico-démographique et sociale). Elle a anticipé sur la décision prise, deux ans plus tard, d'évacuer les anciens déportés vers la Pologne, mais l'a amplement configurée par sa dimension opératoire, montrant ainsi le rôle décisif du système d'enregistrement dans la formulation des actions engagées au sommet de l'État.

Conçus en 1945, les départs massifs des «anciens citoyens polonais » ont surtout résulté de l'enchaînement de différentes logiques de déplacements : mise en mouvement de l'armée Berling, retour vers l'Ouest des évacués de 1941 et transferts des populations frontalières à visée d'homogénéisation ethnique. Le principe de « désoviétisation », théoriquement basé sur l'appartenance ethnique polonaise et juive a, en réalité, reposé sur l'ancienne sujétion polonaise. Comme en 1943, l'action de dépasseportisation s'est présentée sous un double volet. Elle fut d'une part une opération de contrôle administratif, visant à vérifier, à l'instar de la procé- 
dure d'amnistie de 1941, que les candidats au départ correspondent bien à la catégorie des «anciens citoyens polonais », et non à des Soviétiques de nationalité ethnique polonaise. Elle fut, d'autre part, une opération de contrôle politique, dans laquelle la carte de l'Union des patriotes polonais semble avoir pleinement joué son rôle et qui doit être envisagée dans le contexte des retours vers un pays en voie de satellisation. De ce point de vue, l'attestation de loyauté, de même que la constitution de dossiers personnels, est apparue comme l'expression d'un maintien sous surveillance, du moins potentiel. Il reste dès lors à s'interroger sur la « soviétisation » effective des anciens déportés, après six ans de tribulations et de confrontation au système soviétique. Mais l'histoire «polonaise » de ce groupe, hormis les trajectoires de quelques figures politiques comme celle de Jaruzelski, reste encore largement à écrire.

Centre d'études du monde russe, soviétique et post-soviétique EHESS

54, boulevard Raspail

75006 Paris

gousseff@ehess.fr 